Article

\title{
Geochemistry and Origin of Scheelites from the Xiaoyao Tungsten Skarn Deposit in the Jiangnan Tungsten Belt, SE China
}

\author{
Qiangwei Su ${ }^{1, *}$, Jingwen Mao ${ }^{1}$, Jia Sun ${ }^{1}$, Linghao Zhao ${ }^{2}$ and Shengfa $\mathrm{Xu}^{3}$ \\ 1 MLR Key Laboratory of Metallogeny and Mineral Assessment, Institute of Mineral Resources, Chinese \\ Academy of Geological Sciences, Beijing 100037, China; jingwenmao@263.net (J.M.); \\ sunjia.china@yahoo.com (J.S.) \\ 2 National Research Centre for Geoanalysis, Beijing 100037, China; linghaozhao@126.com \\ 3332 Geological Team of Anhui Bureau of Geology and Mineral Resources, Huangshan 245000, China; \\ xushengfa126@126.com \\ * Correspondence: 2001130377@cugb.edu.cn
}

Received: 13 January 2020; Accepted: 11 March 2020; Published: 18 March 2020

\begin{abstract}
The type, association, variations, and valence states of several metal elements of scheelite can trace the source and evolution of the ore-forming fluids. There are four types of scheelite from the Xiaoyao deposit: (1) scheelite intergrown with garnet in the proximal zone (Sch1a) and with pyroxene in the distal zone (Sch1b), (2) scheelite replaced Sch1a (Sch2a) and crystallized as rims around Sch1b (Sch2b), (3) quartz vein scheelite with oscillatory zoning (Sch3), and 4) scheelite (Sch4) within micro-fractures of Sch3. Substitutions involving Mo and $\mathrm{Cd}$ are of particular relevance, and both elements are redox-sensitive and oxidized Sch1a, Sch2b, Sch3 are Mo and Cd enriched, relatively reduced Sch1b, Sch2a, Sch4 are depleted Mo and Cd. Sch1a, Sch2a, Sch3, and Sch4 are characterized by a typical right-inclined rare earth element (REE) pattern, inherited from ore-related granodiorite and modified by the precipitation of skarn minerals. Sch $1 b$ and Sch $2 b$ are characterized by low light rare earth element/heavy rare earth element (LREE/HREE) ratios, influenced by a shift in $\mathrm{fO}_{2}$ during fluid-rock alteration. Sch1b, Sch2b and Sch3 have higher Sr contents than those of Sch1a and Sch2a, reveal that host-rock alteration and fluid-rock interaction have elevated $\mathrm{Sr}$ contents. The Y/Ho ratios of scheelite gradually increase from skarn to quartz vein stages, due to fluid fractionation caused by fluid-rock interaction. Thus, the variation in REE and trace elements in scheelite in time and space reflects a complex magmatic-hydrothermal process involving various fluid-rock interactions and fluid mixing.
\end{abstract}

Keywords: skarn system; scheelite; texture; REE; redox state

\section{Introduction}

Scheelite $\left(\mathrm{CaWO}_{4}\right)$ is one of the dominant tungsten-containing minerals in porphyry-skarn $\mathrm{W}$ deposits, quartz vein $\mathrm{W}$ and $\mathrm{Au}-\mathrm{W}$ deposits, and some metamorphic Au deposits [1,2]. Scheelite incorporates relatively high amounts of REE and Sr in substitution for Ca [3,4], and has been used to characterize the ore-forming fluids [5-8] and the genetic type of deposits [9-11]. The REE patterns in scheelite are highly sensitive to the hydrothermal evolution, making scheelite act as a promising tool to understand prevailing hydrothermal conditions [3,4]. Despite the extensive literature on trace elements in scheelite, only a few studies focus on tungsten skarn deposits [12-15]. Most tungsten skarn deposits are characterized by multiple scheelite generations which underwent dissolution and re-precipitation processes [16-18]. The superimposition of multiple scheelite generations complicates study on the ore-forming processes via the trace element chemistry of scheelite. Cathodoluminescence 
(CL) imaging has been proven to be an effective technique to reveal internal textures, zoning and trace element distributions in scheelite [3]. However, previous studies did not evaluate dissolution and re-precipitation processes in scheelite from tungsten skarn deposits due to the changing hydrothermal fluids $[13,14]$. The detailed textural characterization and systematic investigations of scheelite from each stage prior to in situ chemical analysis are critical to constraint the evolution of skarn systems and W ore precipitation.

The Jiangnan tungsten belt in the southeastern margin of the Yangtze Craton is a new giant tungsten province with $\mathrm{WO}_{3}$ reserves over 5.0 million tons [19-23], including the Dahutang (reserves of $1.10 \mathrm{Mt}$ and grade of $0.18 \%$ ) and the Zhuxi (reserves of $3.44 \mathrm{Mt}$ and grade of $0.54 \%$ ) deposits, as well as other large-scale tungsten deposits, such as Yangchuling, Xiaoyao, Dongyuan, Zhuxiling deposits [24-26]. The present study focuses on the Xiaoyao tungsten skarn deposit, characterized by complex scheelite textural features. The Xiaoyao deposit has reserves of 75,000 tons of $\mathrm{WO}_{3}$ with an average grade of $0.2 \%$ [27]. We present both textural and compositional features for scheelite from the Xiaoyao deposit to evaluate the importance of dissolution and re-precipitation processes in the formation of skarn-related scheelite.

\section{Geologic Setting}

The Xiaoyao tungsten deposit is located in the southeastern part of the Jiangnan tungsten belt (JNB) (Figure 1). The rocks in the mine area are the early Neoproterozoic Xiuning Formation sandstone, late Neoproterozoic Lantian Formation limestone and slate, Piyuancun Formation clastic siliceous rocks, Cambrian Hetang Formation shale, and Yangliugang Formation shale (Figure 2). Most tungsten orebodies are hosted in the Lantian and Hetang Formations.

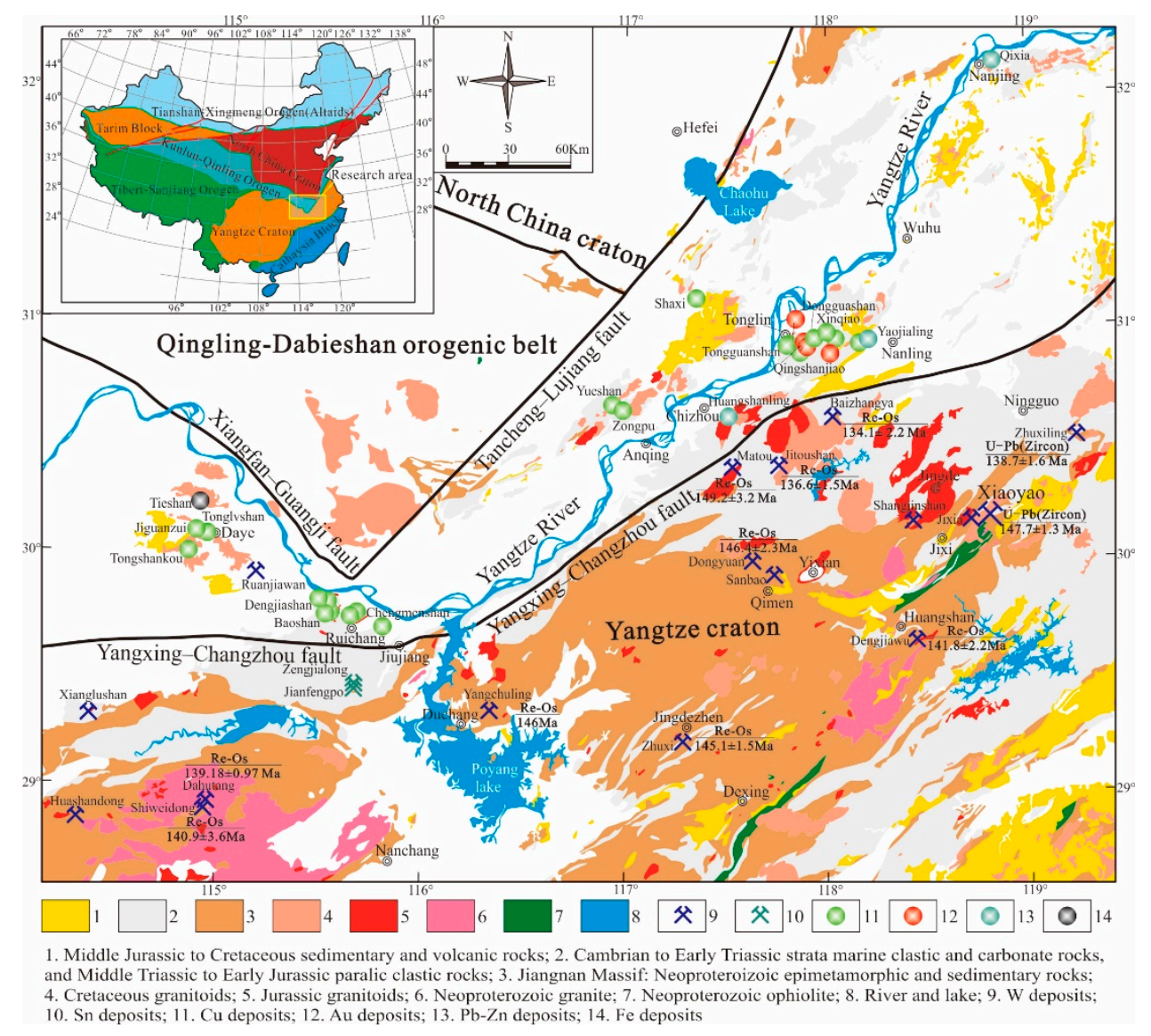

Figure 1. Distribution of the Middle-Lower Yangtze River Valley porphyry-skarn Cu-Au-Mo-Fe ore belt at north and the Jiangnan porphyry-skarn tungsten belt at the south [21]. 
The granitic intrusion in the Xiaoyao mine area comprises granodiorite, granite, and granite porphyry (Figure 2). The granodiorite has a U-Pb zircon age of 149.4 $\pm 1.1 \mathrm{Ma}$ [28]. The granodiorite consists mainly of plagioclase (50-55\%), K-feldspar (10-15\%), quartz (20-25\%), biotite (15-20\%), and hornblende $(3 \%-5 \%)$, with minor apatite, zircon, magnetite, and titanite. The granite porphyry with the $\mathrm{U}-\mathrm{Pb}$ zircon ages of $133.2 \pm 0.8 \mathrm{Ma}$ are younger and crosscuts the granodiorite bodies. Phenocrysts include K-feldspar (20-25\%), plagioclase (10-15\%), and quartz (5-10\%). The granite porphyry matrix is composed of microgranular quartz, plagioclase, and K-feldspar. The granite in the northern part of the mining area was dated to 130-124 Ma by LA-ICP-MS zircon U-Pb methods [29]. This granite contains K-feldspar (45-50\%), quartz (30-35\%), plagioclase (10-15\%), and biotite (2-3\%).

Tungsten orebodies mainly occur within skarns at contact zones between the Kaobeijian granodiorite pluton and their wall rocks (Figure 2). The largest tungsten orebody (W1) developed along the contact between granodiorite and the limestone of the Lantian Formation (Figure 3). It has a length of $1300 \mathrm{~m}$ and a width of 5-35 m. It strikes in an NE direction and dips toward the north at an angle of $80^{\circ}$ (Figure 3).

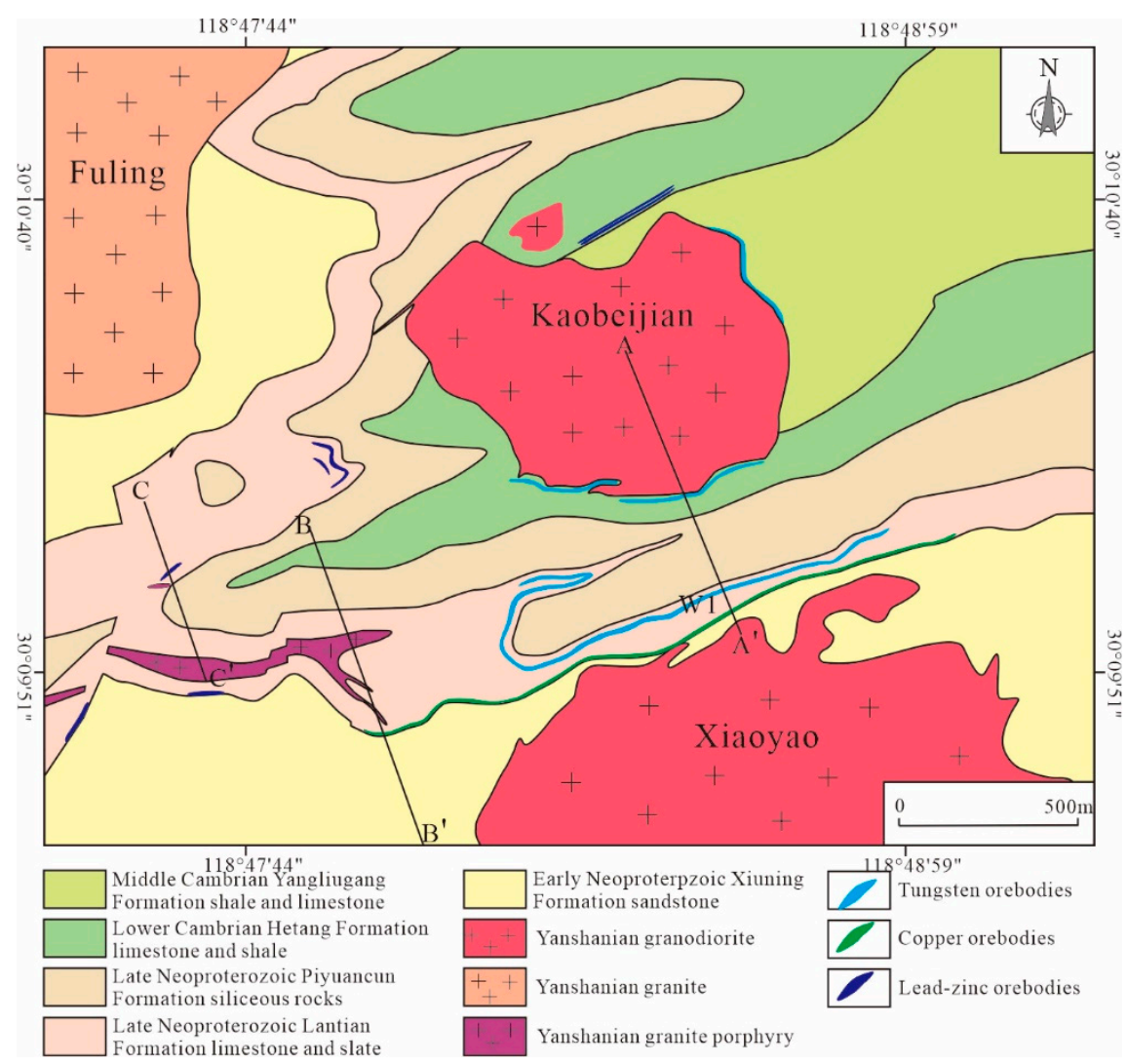

Figure 2. Geological map of the Xiaoyao tungsten deposit [27].

The Xiaoyao skarn is mainly composed of garnet and pyroxene, with retrograde alteration minerals of epidote, tremolite, actinolite, chlorite, quartz, fluorite and calcite. The main ore minerals are scheelite, molybdenite, chalcopyrite, and pyrrhotite. Four mineralization stages have been identified based on crosscutting relationships and mineral assemblage: skarn, retrograde alteration, quartz-scheelite-sulfide, and carbonate stages. 


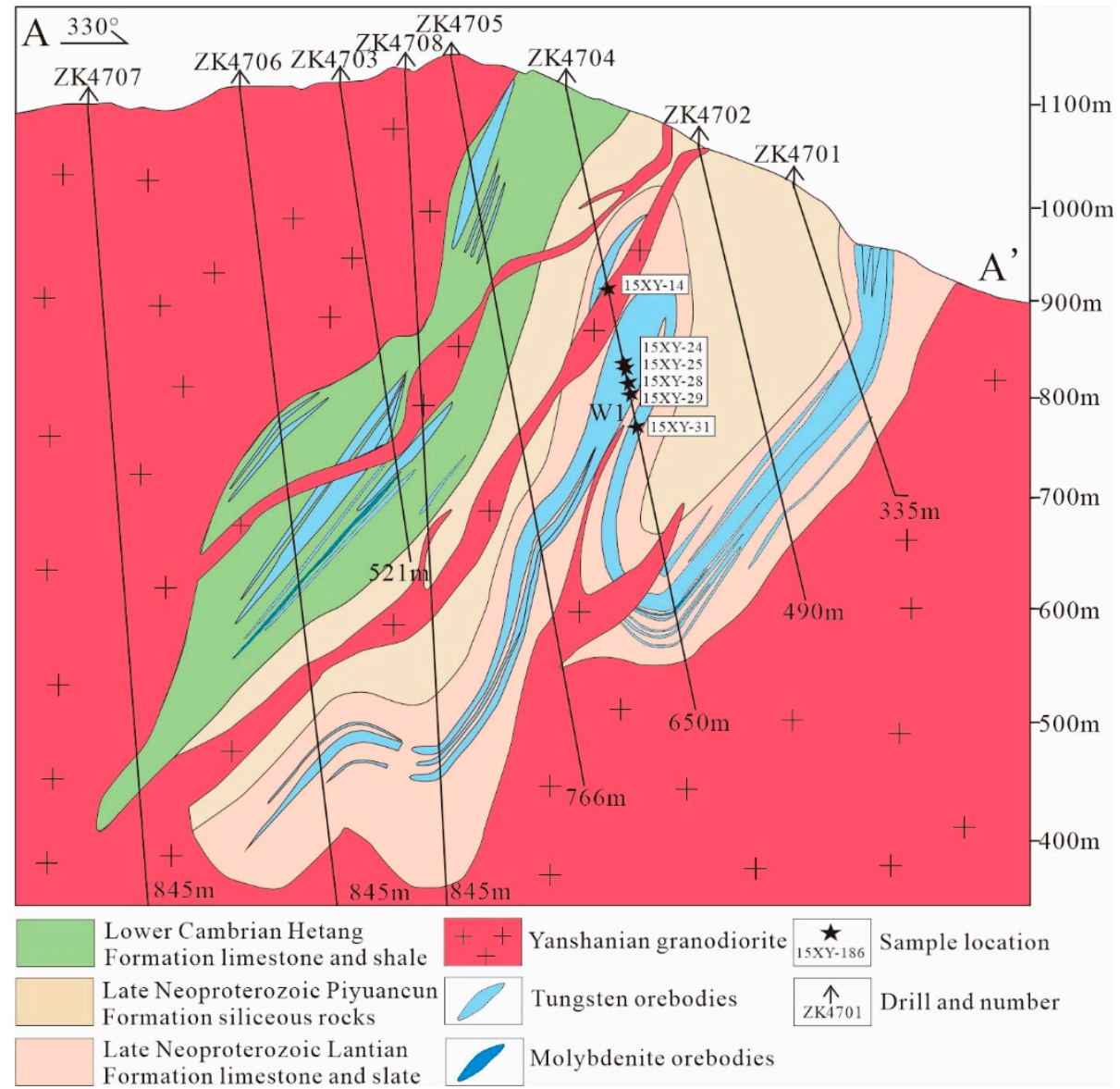

Figure 3. Geological cross-section along exploration No. 47 (A-A' in Figure 2) of the Xiaoyao tungsten deposit [27].

The skarn stage is characterized by a large amount of garnet and pyroxene distributed in different zones (Figure 4a,b). For example, in drill core ZK4704, the skarn has a pyroxene-dominated distal zone and a garnet-dominated proximal zone from shallow to deep. The garnets in proximal zones are always reddish to red and fine- to coarse-grained and euhedral to subhedral with oscillatory zonation (Figure $4 \mathrm{a}$, sample 15XY-31). They commonly intergrow with pyroxenes. The pyroxenes are generally pale green in color. They occur as fine-grained $(0.2-1.0 \mathrm{~mm})$ subhedral to anhedral minerals. The distal zones are dominated by dark green, coarse-grained pyroxene crystals without garnet (Figure $4 \mathrm{~b}$, sample 15XY-14). The pyroxene grains are anhedral to subhedral, varying from 100 to $500 \mu \mathrm{m}$ in size. The retrograde alteration stage is characterized by the precipitation of hydrous silicate minerals such as epidote, actinolite, and chlorite; and the associated mineralization is scheelite replacing garnet and pyroxene (Figure 4c). The quartz-scheelite-sulfide stage is characterized by a scheelite-molybdenite-pyrrhotite-chalcopyrite-quartz assemblage in scheelite- and sulfide-rich veins cutting the early scheelite-bearing skarn ores (Figure $4 \mathrm{~d}-\mathrm{f}$ ). The scheelite in this stage generally occurs as large anhedral grains that intergrow with quartz. The carbonate stage is characterized by a calcite-sphalerite-galena assemblage in sulfide-rich veins that cut through the tungsten ores and wall rock. 

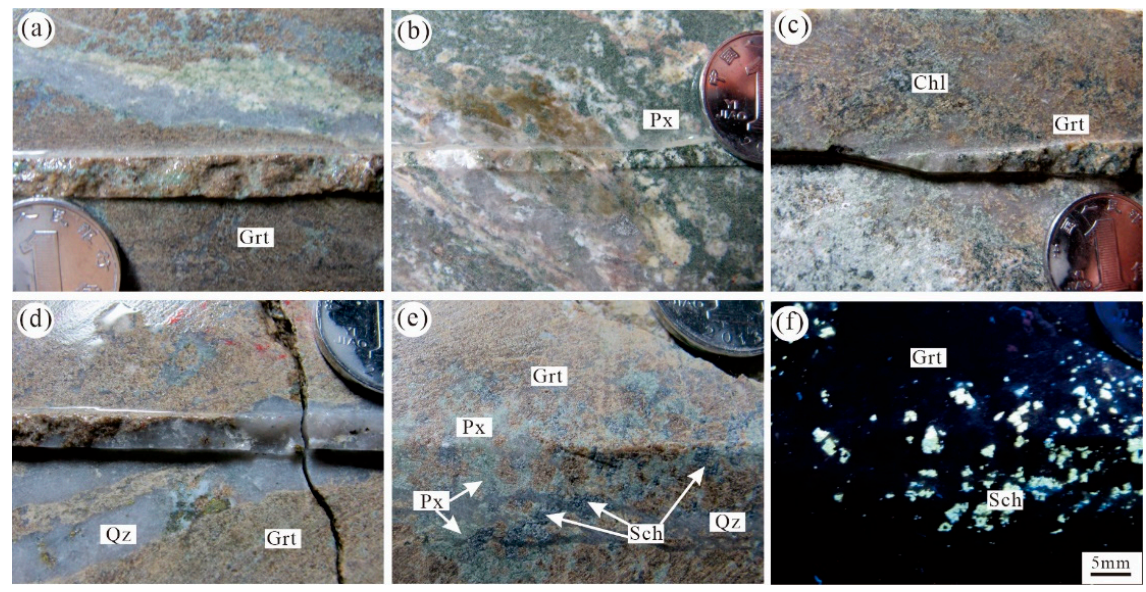

Figure 4. Photographs of tungsten ores. (a) Garnet skarn with disseminated scheelite; (b) Pyroxene skarn with disseminated scheelites; (c) Retrograde alteration skarn; (d) Quartz-scheelite-sulfides vein cutting the garnet skarn; (e) Quartz vein scheelite replaced pyroxene and garnet; (f) Quartz vein scheelite with blue luminescence under ultraviolet light. Grt—garnet; Px—pyroxene; Chl—chlorite; Sch—scheelite; Qz-quartz.

\section{Samples and Analytical Methods}

\subsection{Sample Descriptions}

The samples for electron microprobe and in-situ LA-ICP-MS trace elements analysis were collected from the drill cores taken from the largest W1 orebody (Figure 3). The mineralogical and petrographic features and sampling depth of the samples are summarized in Table 1. Samples were evaluated petrographically employing optical and CL microscopy to characterize the abundance and physical properties of scheelite, including shape, size, color, and luminescence. Selected grains were analyzed by electron probe microanalysis (EPMA) for major element geochemistry and in situ LA-ICP-MS for trace element geochemistry.

Based on CL textures and mineral paragenesis, four types of scheelite have been recognized. Scheelite 1 (Sch1) occurs as disseminated crystal in the skarn (Figure $5 a, e$ ), which is further divided based on different pyroxene, garnet and scheelite assemblages. Scheelite 1a (Sch1a) is intergrown with garnet in the proximal zone (Figure $5 \mathrm{a}$ ) and Scheelite $1 \mathrm{~b}(\mathrm{Sch} 1 \mathrm{~b})$ is intergrown with pyroxene in the distal zone (Figure 5e). Sch1a shows yellowish fluorescent in short-waved UV light. It is mainly fine-grained, euhedral to subhedral, with diameters of 0.1 to $0.2 \mathrm{~mm}$ (Figure 5a). Sch1a shows homogeneous textures with dark-CL (weak CL response, Figure 5b), reflecting that no dissolution took place during any stage of growth. Sch1b shows blue fluorescent in short-waved UV light and generally occurs as anhedral coarse grains of $0.5-1.0 \mathrm{~mm}$ in size (Figure 5e). The homogeneous textures, as shown by the strong and bright CL of Sch1b, may be precipitation features associated with precipitating under stable conditions (Figure 5f). Scheelite 2a (Sch2a) selectively replaced parts of Sch1a that likely represent relict fluid pathways during the alteration stage (Figure $5 c, h$ ). Sch2a occurs as irregular, with a light-CL with a grain size of $0.2-0.5 \mathrm{~mm}$ (Figure $5 \mathrm{c}, \mathrm{h}$ ). The Sch2a crystal shows a strong correlation with molybdenite (Figure $5 \mathrm{~d}$ ) and is often associated with epidote, actinolite, chlorite, and quartz (Figure $5 g$ ). Scheelite $2 b(S c h 2 b)$ is crystallized as rims around the Sch1b with dark-CL (Figure 5f). Sch2b generally occurs as anhedral coarse grain of 1.0-1.5 mm in size (Figure 5e). CL images of Sch2a and Sch $2 b$ from the mineralized skarn reveal later dissolution-reprecipitation reactions (Figure $5 \mathrm{c}, \mathrm{f}, \mathrm{h}$ ).

Scheelite 3 (Sch3) forms abundant large scheelite clusters within scheelite \pm sulfide-rich quartz veins that typically crosscut the early scheelite-bearing skarn ores (Figure 51). Sch3 generally occurs as a subhedral-anhedral coarse grain of $0.2-1.5 \mathrm{~mm}$ in size (Figure 5l). Sch3 exhibit obvious oscillatory zoning textures, with a few examples showing the core-rim textures on CL images (Figure 5j,k). The rim is oscillatory growth zoning and the core is homogeneous, as shown on CL images (Figure 5j,k). 
Scheelite 4 (Sch4) occurs as rims around Sch3 (Figure 5j) or as micro-fractures within Sch3 with light-CL (Figure 5k). The occurrence of micro-fractures in Sch3, which are filled with Sch4, indicates recrystallization during late hydrothermal processes. The formation of Sch4 is accompanied by the precipitation of molybdenite and other sulfides (chalcopyrite, pyrrhotite; Figure 5l).

Table 1. Details of scheelite samples from the Xiaoyao deposit.

\begin{tabular}{cccc}
\hline Sample & Scheelite Type & Location & Sample Description \\
\hline 15XY-31 & Sch1a, 2a & $353 \mathrm{~m}$, ZK4704 & Proximal skarn tungsten ore \\
$15 X Y-14$ & Sch1b, 2b & $208 \mathrm{~m}$, ZK4704 & Distal skarn tungsten ore \\
$15 \mathrm{XY}-28$ & Sch1a, 2a & $313 \mathrm{~m}$, ZK4704 & Retrograde skarn tungsten ore \\
$15 \mathrm{XY}-25$ & Sch1a, 3, 4 & $300 \mathrm{~m}$, ZK4704 & Scheelite-rich quartz veins \\
$15 \mathrm{XY}-29$ & Sch3, 4 & $320 \mathrm{~m}, \mathrm{ZK} 4704$ & Scheelite-rich quartz veins \\
$15 \mathrm{XY}-24$ & Sch3, 4 & $290 \mathrm{~m}$, ZK4704 & Scheelite-rich quartz veins \\
\hline
\end{tabular}
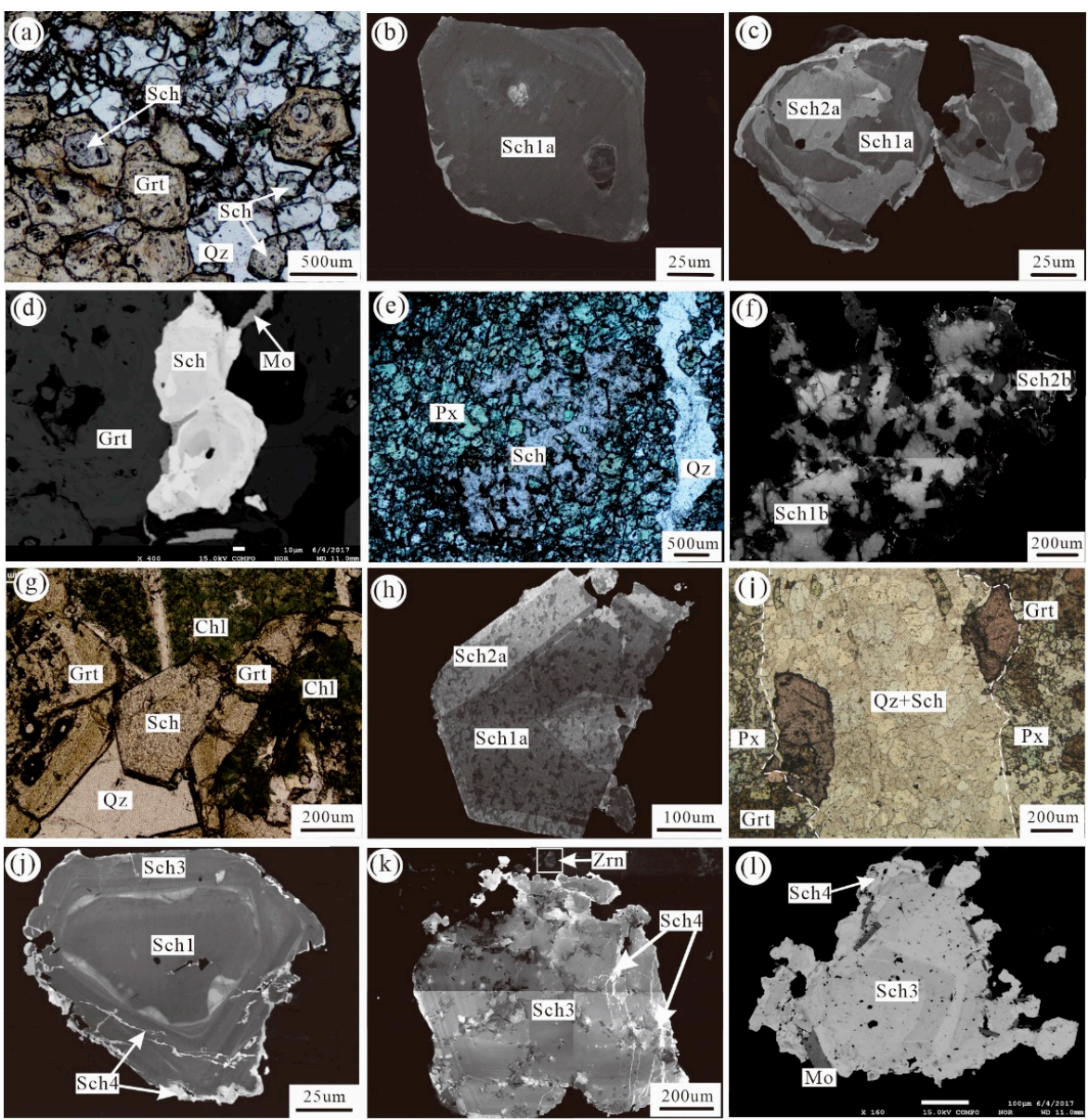

Figure 5. Light, Back scattered Electron (BSE) and Cathodoluminescence (CL) images of scheelites. (a) Sch1a intergrowth with garnet with transmitted light; (b) CL image of Sch1a with homogeneous texture; (c) CL images of Sch1a replaced/overgrowth by Sch2a; (d) BSE images of Sch2a intergrowth with molybdenite; (e) Pyroxene replaced by scheelite with transmitted light; (f) CL images of Sch1b replaced/overgrowth by Sch2b; (g) Retrograde alteration stage scheelite with transmitted light; (h) CL images of Sch1a replaced/overgrowth by Sch2a; (i) Transmitted light image of Sch3-rich quartz vein; (j) Core-rim texture revealed by CL image of Sch3 with Sch1, and Sch4 filled within microfractures of Sch3; (k) CL image of Sch4 filled within microfractures of Sch3 with oscillatory growth zoning; (1) BSE image of Sch4 intergrowth with molybdenite. Sch—scheelite; Mo-molybdenite; Grt-garnet; Px-pyroxene; Chl—chlorite; Qz-quartz; Zrn—zircon. 


\subsection{Cathodoluminescence}

Cathodoluminescence is an efficient technique for determining the zonation patterns and growth history of scheelite which are otherwise not unobservable using light microscopy. CL imaging of scheelite was obtained using a Gatan MonoCL 4 system attached to the FEI Nova NanoSEM 450 scanning electron microscope (FEI corporation, Hillsboro, OR, USA) at $10 \mathrm{kV}$, at the Institute of Geology, Chinese Academy of Geological Sciences, Beijing.

\subsection{Electron Microprobe Analysis}

Chemical compositions of scheelite were determined by electron probe microanalyses using a JEOL JXA-8230 Superprobe (JOEL Ltd., Akashima, Tokyo, Japan) at the Institute of Mineral Resources, Chinese Academy of Geological Sciences, Beijing. The operating conditions were an accelerating voltage of $15 \mathrm{kV}$, a beam current of $10 \mathrm{nA}$, and a count time per element of $10 \mathrm{~s}$ for major elements. The quantitative analyses were performed with carbon-coated thin sections in an evacuated chamber with less than $4.0 \mathrm{ppm}$ mbar. The following natural minerals and synthetic oxides were used for calibration: $\mathrm{K}$-fedspar $(\mathrm{K})$, jadeite $(\mathrm{Na})$, apatite $(\mathrm{P})$, wollastonite $(\mathrm{Ca})$, hematite $(\mathrm{Fe}), \mathrm{MnO}(\mathrm{Mn})$, scheelite (W), $\mathrm{Cr}_{2} \mathrm{O}_{3}(\mathrm{Cr})$, wulfenite (Mo), and rutite (Ti).

\subsection{In situ LA-ICP-MS Trace Element Analysis}

Trace element concentrations were determined by laser ablation-inductively coupled plasma-mass spectrometry (LA-ICP-MS) using a New Wave UP 213 laser ablation system coupled to a Thermo Element II single collector high-resolution magnetic sector (ICP-MS) at the National Research Center for Geoanalysis, Beijing, China. Helium is advantageous as a carrier gas and was thus applied in this study. Single-spot analyses were performed by laser-ablating spot diameters of $40 \mu \mathrm{m}$ with a repetition rate of $10 \mathrm{~Hz}$ at an energy density of $23-25 \mathrm{~J} / \mathrm{cm}^{2}$. Each LA-ICP-MS analysis incorporated a $\sim 20 \mathrm{~s}$ background acquisition (gas blank) followed by a $60 \mathrm{~s}$ data acquisition from the sample. After every ten spots, analysis was followed by one NIST SRM 610 analysis to correct the time-dependent drift in sensitivity and mass discrimination of the ICP-MS. Reference glasses (GSE, NIST612, NIST614) were analyzed prior to and after the sample measurements. Calcium 44 was used as an internal standard and fixed at $19.55 \mathrm{wt}$ \%, based on the average EMPA composition.

\section{Analytical Results}

\subsection{Major Element Geochemistry}

Microprobe analyses of scheelite from the Xiaoyao tungsten deposit are shown in Table 2. The scheelite at Xiaoyao has a wide range of $\mathrm{WO}_{3}(66.67-80.45 \%), \mathrm{MoO}_{3}(0.19-10.30 \%)$ contents, and $\mathrm{CaO}$ concentrations of $18.70-20.97 \%$ (Table 2 and Figure 6a). Sch1a in the proximal zone has low $\mathrm{WO}_{3}$ (68.95-73.87\%) and $\mathrm{CaO}(18.75-20.97 \%)$ and high $\mathrm{MoO}_{3}(5.77-10.30 \%)$ contents, while Sch1b in the distal zone has high $\mathrm{WO}_{3}(79.78-79.88 \%)$ and $\mathrm{CaO}(19.51-19.59 \%)$ and low $\mathrm{MoO}_{3}(0.38-0.42 \%)$ contents. In the retrograde alteration stage, Sch2a has relatively high $\mathrm{WO}_{3}(76.59-78.13 \%)$ and $\mathrm{CaO}(18.70 \%-19.70 \%)$, and relatively low $\mathrm{MoO}_{3}(1.71-3.51 \%)$ contents, while Sch2b has low $\mathrm{WO}_{3}(70.03 \%-70.71 \%)$ and $\mathrm{CaO}$ (19.25-20.93\%), and high $\mathrm{MoO}_{3}(8.06-10.17 \%)$ contents. In the quartz-scheelite-sulfide stage, Sch3 has relatively low $\mathrm{WO}_{3}(66.67-74.55 \%)$ and $\mathrm{CaO}(19.00-20.83 \%)$, and relatively high $\mathrm{MoO}_{3}(4.09-9.78 \%)$ contents. Sch4 has relatively high $\mathrm{WO}_{3}(79.13-80.48 \%)$ and $\mathrm{CaO}(18.73-19.70 \%)$, and relatively low $\mathrm{MoO}_{3}(0.19-0.76 \%)$ contents (Table 2 and Figure 6a). 
Table 2. Major elements of four types of scheelites from the Xiaoyao deposit (\%).

\begin{tabular}{|c|c|c|c|c|c|c|c|c|c|c|}
\hline No. & Type & $\mathrm{WO}_{3}$ & $\mathrm{P}_{2} \mathrm{O}_{5}$ & $\mathrm{CaO}$ & $\mathrm{FeO}$ & $\mathrm{MnO}$ & $\mathrm{V}_{2} \mathrm{O}_{3}$ & $\mathrm{TiO}_{2}$ & $\mathrm{MoO}_{3}$ & Total \\
\hline 15XY-31-1-1 & Sch1a & 72.41 & 0.03 & 20.85 & 0.02 & 0 & 0 & 0.02 & 5.77 & 99.11 \\
\hline $15 X Y-31-1-2$ & Sch1a & 71.06 & 0.05 & 20.97 & 0.07 & 0.02 & 0.02 & 0.01 & 7.26 & 99.49 \\
\hline $15 X Y-31-1-3$ & Sch1a & 68.95 & 0 & 19.99 & 0 & 0 & 0.02 & 0.02 & 10.3 & 99.32 \\
\hline $15 X Y-31-1-4$ & Sch1a & 69.38 & 0.1 & 20.26 & 0.04 & 0 & 0 & 0.01 & 10.23 & 100.06 \\
\hline $15 X Y-31-1-5$ & Sch1a & 72.03 & 0.04 & 20.86 & 0 & 0 & 0.01 & 0 & 7.05 & 100.05 \\
\hline $15 X Y-31-1-6$ & Sch2a & 77.76 & 0.02 & 19.7 & 0.17 & 0.03 & 0.09 & 0.03 & 1.71 & 99.53 \\
\hline $15 X Y-28-2-5$ & Sch2a & 76.59 & 0.06 & 18.89 & 0 & 0 & 0 & 0.02 & 3.51 & 99.1 \\
\hline $15 X Y-28-2-6$ & Sch1a & 71.23 & 0.02 & 19.04 & 0.02 & 0 & 0.04 & 0.02 & 8.7 & 99.12 \\
\hline $15 X Y-28-2-7$ & Sch1a & 73.87 & 0.03 & 18.75 & 0.01 & 0 & 0 & 0 & 8.03 & 100.76 \\
\hline $15 X Y-28-2-8$ & Sch2a & 78.13 & 0.08 & 18.7 & 0 & 0.01 & 0.01 & 0 & 2.14 & 99.12 \\
\hline 15XY14-3-1 & Sch1b & 79.79 & 0 & 19.59 & 0 & 0 & 0 & 0 & 0.42 & 99.84 \\
\hline 15XY14-3-2 & Sch2b & 70.71 & 0.04 & 19.25 & 0 & 0 & 0 & 0 & 10.17 & 100.22 \\
\hline 15XY14-3-3 & Sch2b & 70.03 & 0.05 & 20.93 & 0.03 & 0 & 0.04 & 0.02 & 8.07 & 99.2 \\
\hline 15XY14-3-4 & Sch1b & 79.88 & 0.01 & 19.51 & 0 & 0 & 0 & 0 & 0.38 & 99.86 \\
\hline $15 X Y-25-2-1$ & Sch1a & 69.79 & 0.11 & 20.06 & 0 & 0 & 0.08 & 0.01 & 9.34 & 99.46 \\
\hline $15 X Y-25-2-2$ & Sch1a & 73.79 & 0 & 18.95 & 0 & 0.01 & 0 & 0 & 7.61 & 100.39 \\
\hline $15 X Y-25-2-3$ & Sch3 & 69.28 & 0.05 & 20.09 & 0 & 0.02 & 0 & 0 & 9.78 & 99.23 \\
\hline $15 X Y-25-2-4$ & Sch3 & 66.67 & 0.05 & 20.36 & 0.1 & 0.04 & 0 & 0 & 8.98 & 96.19 \\
\hline $15 X Y-25-2-5$ & Sch4 & 79.8 & 0.06 & 19.44 & 0.02 & 0.03 & 0 & 0.01 & 0.33 & 99.7 \\
\hline $15 X Y-25-2-6$ & Sch1a & 71.29 & 0.07 & 20.25 & 0.07 & 0 & 0.04 & 0 & 8.13 & 99.87 \\
\hline $15 X Y-25-2-7$ & Sch3 & 71.44 & 0.09 & 20.52 & 0 & 0.04 & 0.03 & 0.02 & 6.88 & 99.01 \\
\hline 15XY29-3-1 & Sch3 & 72.53 & 0.04 & 20.83 & 0 & 0 & 0 & 0.01 & 6.61 & 100.06 \\
\hline 15XY29-3-2 & Sch3 & 74.43 & 0.02 & 19.09 & 0 & 0.07 & 0.03 & 0.03 & 5.63 & 99.32 \\
\hline 15XY29-3-3 & Sch3 & 74.55 & 0.09 & 20.24 & 0 & 0 & 0.03 & 0.01 & 4.09 & 99.01 \\
\hline 15XY29-3-4 & Sch4 & 79.13 & 0.08 & 19.7 & 0.22 & 0.05 & 0 & 0 & 0.76 & 99.95 \\
\hline $15 X Y-24-2-1$ & Sch3 & 72.15 & 0.02 & 19 & 0.04 & 0 & 0 & 0 & 8.05 & 99.29 \\
\hline $15 X Y-24-2-2$ & Sch3 & 72.6 & 0 & 19.07 & 0.01 & 0 & 0 & 0 & 8.17 & 99.95 \\
\hline $15 X Y-24-2-3$ & Sch3 & 71.81 & 0.06 & 19.39 & 0.1 & 0.08 & 0 & 0 & 9.37 & 100.83 \\
\hline $15 X Y-24-2-4$ & Sch3 & 71.83 & 0 & 19 & 0 & 0.03 & 0 & 0.01 & 9.49 & 100.46 \\
\hline $15 X Y-24-2-5$ & Sch3 & 71.58 & 0.08 & 19.07 & 0.03 & 0 & 0 & 0 & 9.35 & 100.15 \\
\hline $15 X Y-24-2-6$ & Sch3 & 72.66 & 0.01 & 19.02 & 0.07 & 0.02 & 0 & 0 & 9.06 & 100.88 \\
\hline $15 X Y-24-2-7$ & Sch4 & 80.48 & 0.02 & 18.73 & 0 & 0 & 0 & 0 & 0.19 & 99.44 \\
\hline
\end{tabular}
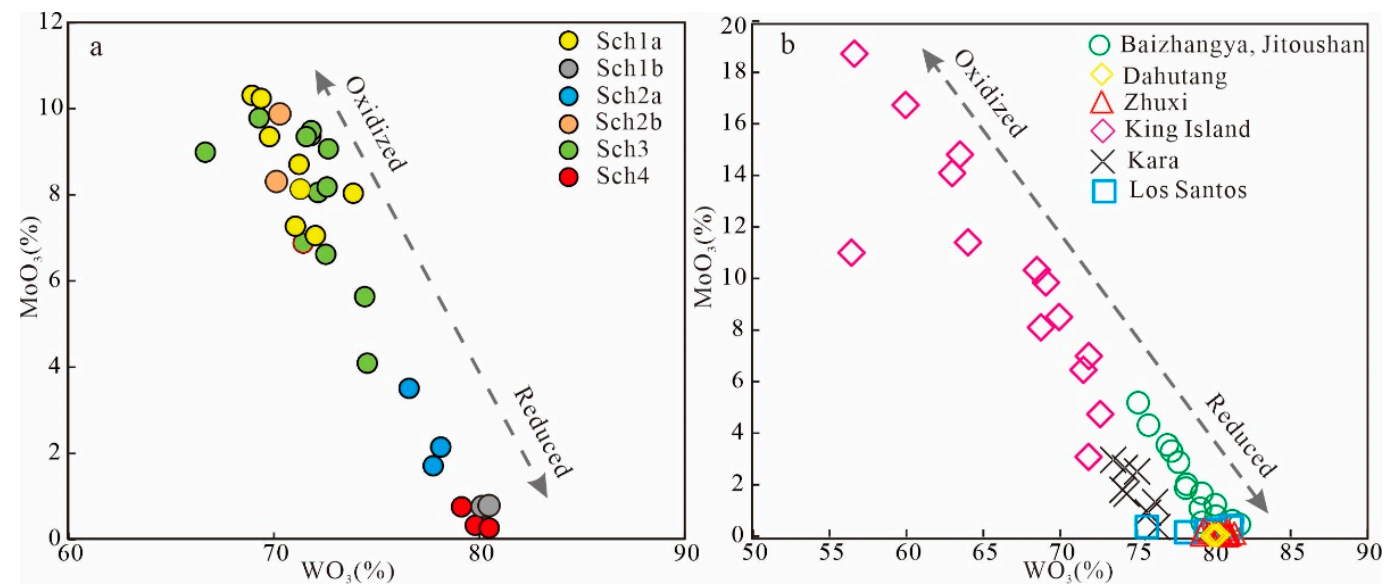

Figure 6. Plots of $\mathrm{WO}_{3}$ vs. $\mathrm{MoO}_{3}$ of Xiaoyao scheelites (a) and scheelites from other porphyry-skarn tungsten deposits are shown for comparison (b), including the Dahutang tungsten deposit [30], the Jitoushan and Baizhangya tungsten deposits [9], the King-Island-oxidized skarn deposit [17], the Kara-oxidized skarn deposit [31], and the Los-Santos-reduced skarn deposit [32].

\subsection{Trace Element Geochemistry.}

The representative trace element analyses from LA-ICP-MS on garnet, pyroxene and scheelite are listed in Table 3; and the chondrite-normalized REE patterns are shown in Figure 7.

Garnet is characterized by a slight LREE enrichment relative to HREE with low LREE/HREE ratios of 1.4-3.0 (Figure 7a,b), and the total REE contents are 85.9-91.0 ppm, accompanied by Eu/Eu* values 
of 0.57-0.62. In addition, garnet has $W$ contents of 219-384 ppm, Mo contents of 4.2-7.4 ppm, and $\mathrm{Y}$ contents of 15.7-33.2 ppm. Pyroxene is characterized by HREE enrichment relative to LREE, with low LREE/HREE ratios of 0.5 (Figure 7c,d), and the total REE contents are 8.1-8.2 ppm, accompanied by varying $\mathrm{Eu} / \mathrm{Eu}^{*}$ values of $0.56-2.41$. In addition, pyroxene has $\mathrm{Sr}$ contents of 7.4-11.7 ppm and Y contents of 4.9-5.0 ppm.

Sch1a is characterized by a typical right-inclined REE pattern with high LREE/HREE ratios of 16.9-41.0 (Figure 7a,b), and the total REE contents are 93.8-280.2 ppm, accompanied by the lowest $\mathrm{Eu} / \mathrm{Eu}^{*}$ values of $0.22-0.33$. In addition, Sch1a has $\mathrm{Sr}$ contents of $67.0-87.1 \mathrm{ppm}, \mathrm{Y}$ contents of 2.0-3.5 ppm. As contents of 3.3-11.0 ppm, and high Ta contents of 779.4-863.9 ppm. Sch1b is characterized by an HREE-enrichment pattern with low LREE/HREE ratios of 0.3-0.7 (Figure 7c,d), and the total REE contents are 237.3-454.4 ppm, accompanied by Eu/Eu* values of 0.34-0.57. The Sr contents of 76.7-110.2 ppm, Y contents of 143.3-496.8 ppm. As contents of 4.3-12.9 ppm, Ta contents of 1397.2-1568.9 ppm in the Sch1b are generally higher than those of Sch1a.

Sch2a displays the same pattern, with high LREE/HREE ratios of 16.0-47.8, lower total REE contents of 21.2-186.5 ppm, and higher Eu/Eu* values of 0.50-1.09, compared with Sch1a (Figure 7a,b). The Y contents of 0.4-1.9 ppm and Y/Ho values of 13.78-20.43 in Sch2a are lower than those of Sch1a. However, Sch2a has higher Sr contents of 73.2-89.7 ppm and Ta contents of 860.2-928.0 ppm. Sch2b is characterized by an LREE-enrichment pattern with low LREE/HREE ratios of 0.7-2.1 (Figure 7c,d) and the total REE contents are $256.7-600.8 \mathrm{ppm}$, accompanied by Eu/Eu* values of $0.50-0.77$. In addition, Sch2b has higher Sr contents of 117.7-159.6 pm and as contents of 6.2-18.5 ppm; however, compared with Sch1b, Sch2b is relatively depleted in Y contents of105.5-195.5 ppm and Ta contents of 1124.9-1359.4 ppm. 
Table 3. Rare earth element and trace element concentrations determined by LA-ICP-MS in four types of scheelite and garnet and pyroxene from the Xiaoyao deposit (ppm).

\begin{tabular}{|c|c|c|c|c|c|c|c|c|c|c|c|c|c|c|c|c|c|c|c|c|c|c|}
\hline Sample & Sch Type & La & $\mathrm{Ce}$ & Pr & Nd & Sm & $\mathrm{Eu}$ & Gd & $\mathrm{Tb}$ & Dy & Ho & Er & $\mathrm{Tm}$ & $\mathrm{Yb}$ & $\mathrm{Lu}$ & $\Sigma$ REE & इLREE & इHREE & LREE/HREE & $\mathrm{Eu} / \mathrm{Eu}^{*}$ & $(\mathrm{La} / \mathrm{Sm})_{\mathrm{N}}$ & $(\mathrm{Gd} / \mathrm{Lu})_{\mathrm{N}}$ \\
\hline $15 X Y-31-1$ & Sch 1a & 67.15 & 95.30 & 8.99 & 26.66 & 2.44 & 0.19 & 1.66 & 0.18 & 0.63 & 0.10 & 0.20 & 0.01 & 0.03 & 0.04 & 203.58 & 200.74 & 4.89 & 41.02 & 0.27 & 17.30 & 6.05 \\
\hline $15 X Y-31-3$ & Sch 1a & 32.09 & 42.05 & 3.64 & 11.69 & 1.50 & 0.15 & 1.21 & 0.12 & 0.70 & 0.13 & 0.25 & 0.02 & 0.18 & 0.07 & 93.80 & 91.13 & 5.39 & 16.92 & 0.33 & 13.44 & 2.33 \\
\hline $15 X Y-31-6$ & Sch 1a & 100.86 & 132.39 & 10.89 & 28.48 & 3.01 & 0.20 & 2.30 & 0.24 & 1.14 & 0.20 & 0.34 & 0.03 & 0.14 & 0.04 & 280.23 & 275.82 & 7.90 & 34.91 & 0.22 & 21.09 & 7.21 \\
\hline $15 X Y-31-2$ & Sch 2a & 3.74 & 11.48 & 1.17 & 3.67 & 0.47 & 0.06 & 0.28 & 0.04 & 0.18 & 0.03 & 0.06 & 0.00 & 0.02 & 0.05 & 21.24 & 20.59 & 1.10 & 18.68 & 0.50 & 4.97 & 0.75 \\
\hline $15 X Y-31-4$ & Sch 2a & 6.67 & 13.41 & 1.60 & 5.68 & 0.44 & 0.16 & 0.69 & 0.07 & 0.22 & 0.04 & 0.09 & 0.00 & 0.01 & 0.04 & 29.10 & 27.95 & 1.75 & 16.01 & 0.88 & 9.62 & 2.48 \\
\hline $15 X Y-31-5$ & Sch $2 a$ & 67.51 & 89.28 & 7.42 & 18.38 & 1.46 & 0.43 & 0.84 & 0.09 & 0.40 & 0.09 & 0.17 & 0.04 & 0.27 & 0.07 & 186.46 & 184.48 & 3.86 & 47.83 & 1.09 & 29.15 & 1.49 \\
\hline $15 X Y-14-1$ & Sch $1 \mathrm{~b}$ & 18.80 & 47.68 & 10.41 & 82.40 & 27.55 & 5.60 & 48.25 & 6.68 & 41.76 & 10.02 & 25.00 & 2.38 & 11.10 & 1.45 & 339.07 & 192.44 & 392.51 & 0.49 & 0.47 & 0.43 & 4.27 \\
\hline $15 X Y-14-2$ & Sch $1 \mathrm{~b}$ & 10.46 & 43.04 & 11.58 & 100.64 & 36.65 & 5.98 & 74.58 & 11.22 & 78.54 & 18.72 & 42.84 & 3.85 & 15.01 & 1.30 & 454.41 & 208.36 & 742.83 & 0.28 & 0.34 & 0.18 & 7.38 \\
\hline $15 X Y-14-3$ & Sch $1 \mathrm{~b}$ & 22.51 & 47.86 & 9.02 & 65.83 & 19.21 & 4.92 & 34.92 & 4.55 & 26.67 & 6.34 & 17.42 & 1.41 & 5.60 & 0.73 & 266.99 & 169.34 & 252.57 & 0.67 & 0.57 & 0.74 & 6.14 \\
\hline $15 X Y-14-8$ & Sch $1 \mathrm{~b}$ & 22.17 & 50.06 & 9.11 & 57.93 & 19.15 & 3.74 & 31.07 & 4.17 & 24.67 & 6.07 & 14.41 & 1.49 & 6.16 & 0.86 & 251.06 & 162.16 & 232.21 & 0.70 & 0.47 & 0.73 & 4.62 \\
\hline 15XY-14-11 & Sch $1 \mathrm{~b}$ & 20.08 & 42.94 & 7.95 & 54.98 & 16.77 & 2.65 & 32.10 & 3.87 & 25.73 & 5.63 & 14.38 & 1.49 & 7.93 & 0.85 & 237.34 & 145.37 & 237.37 & 0.61 & 0.34 & 0.75 & 4.85 \\
\hline $15 X Y-14-6$ & Sch $2 b$ & 24.74 & 52.76 & 9.13 & 60.83 & 16.06 & 3.69 & 30.55 & 4.50 & 25.67 & 6.00 & 14.14 & 1.41 & 6.55 & 0.67 & 256.72 & 167.22 & 234.35 & 0.71 & 0.50 & 0.97 & 5.87 \\
\hline $15 X Y-14-7$ & Sch $2 b$ & 40.06 & 102.91 & 17.54 & 96.14 & 20.94 & 4.69 & 22.11 & 2.68 & 15.36 & 3.06 & 9.00 & 0.86 & 5.87 & 0.56 & 341.78 & 282.29 & 164.99 & 1.71 & 0.66 & 1.20 & 5.04 \\
\hline 15XY-14-9 & Sch $2 b$ & 67.73 & 152.26 & 23.47 & 134.49 & 26.30 & 6.04 & 31.48 & 4.74 & 27.44 & 6.08 & 15.42 & 1.97 & 12.10 & 1.42 & 510.94 & 410.28 & 296.12 & 1.39 & 0.64 & 1.62 & 2.85 \\
\hline $15 X Y-14-10$ & Sch $2 b$ & 69.51 & 151.71 & 23.51 & 117.75 & 20.09 & 5.60 & 24.78 & 3.09 & 15.86 & 3.92 & 10.33 & 1.12 & 6.83 & 0.85 & 454.97 & 388.17 & 185.41 & 2.09 & 0.77 & 2.18 & 3.72 \\
\hline $15 X Y-14-12$ & Sch $2 b$ & 87.42 & 199.19 & 30.75 & 146.76 & 28.05 & 6.91 & 30.70 & 4.28 & 26.68 & 5.96 & 16.53 & 2.11 & 13.95 & 1.53 & 600.82 & 499.06 & 295.67 & 1.69 & 0.72 & 1.96 & 2.57 \\
\hline 15XY-29-2-1 & Sch 3 & 139.81 & 171.97 & 12.56 & 30.53 & 2.44 & 1.05 & 1.48 & 0.15 & 0.75 & 0.11 & 0.27 & 0.02 & 0.21 & 0.07 & 361.43 & 358.37 & 6.41 & 55.87 & 1.56 & 35.98 & 2.71 \\
\hline $15 X Y-29-2-2$ & Sch 3 & 240.02 & 314.24 & 22.89 & 54.09 & 5.66 & 3.83 & 4.82 & 0.56 & 2.66 & 0.47 & 0.70 & 0.08 & 0.32 & 0.04 & 650.38 & 640.73 & 21.28 & 30.11 & 2.19 & 26.67 & 14.12 \\
\hline $15 X Y-29-2-3$ & Sch 3 & 303.46 & 434.53 & 26.56 & 62.70 & 5.40 & 5.55 & 3.77 & 0.37 & 1.91 & 0.26 & 0.30 & 0.07 & 0.10 & 0.05 & 845.03 & 838.19 & $\begin{array}{l}2.20 \\
15.62\end{array}$ & 53.66 & 3.58 & 35.38 & $\begin{array}{l}9.112 \\
9.84\end{array}$ \\
\hline $15 X Y-29-2-4$ & Sch 3 & 298.57 & 434.24 & 23.49 & 49.60 & 3.17 & 3.92 & 1.44 & 0.17 & 0.91 & 0.18 & 0.30 & 0.01 & 0.11 & 0.04 & 816.14 & 812.98 & 8.45 & 96.24 & 4.89 & 59.24 & 4.29 \\
\hline 15XY-29-2-5 & Sch 3 & 248.33 & 290.72 & 16.92 & 33.88 & 2.89 & 1.27 & 1.79 & 0.16 & 0.88 & 0.13 & 0.37 & 0.04 & 0.25 & 0.06 & 597.68 & 594.00 & 9.30 & 63.90 & 1.59 & 54.13 & 4.08 \\
\hline $15 X Y-29-2-6$ & Sch 3 & 208.14 & 222.83 & 16.58 & 42.95 & 4.65 & 1.16 & 3.23 & 0.37 & 2.33 & & 1.09 & 0.12 & & & & & & & & & \\
\hline $15 X Y-29-2-7$ & Sch 3 & 116.48 & 178.67 & 21.28 & 95.76 & 18.06 & 3.57 & 20.30 & 2.41 & 13.77 & 2.52 & 5.40 & 0.48 & 1.45 & 0.14 & 480.28 & 433.82 & 120.94 & 3.59 & 0.57 & 4.06 & 18.20 \\
\hline $15 X Y-29-2-8$ & Sch 3 & 264.81 & 373.44 & 35.97 & 123.47 & 21.39 & 4.75 & 23.86 & 3.41 & 19.29 & 4.42 & 10.28 & 1.02 & 3.65 & 0.35 & 890.13 & 823.84 & 208.97 & & 0.64 & 7.79 & 8.87 \\
\hline $15 X Y-29-2-9$ & Sch 4 & 67.12 & 110.26 & 12.48 & 41.43 & 5.23 & 0.69 & 5.12 & 0.62 & 3.20 & 0.62 & 1.58 & 0.12 & 0.58 & 0.10 & 249.14 & 237.20 & & & & 8.07 & 6.71 \\
\hline $15 X Y-29-2-10$ & Sch 4 & 46.74 & 84.89 & 10.06 & 34.66 & 4.08 & 0.93 & 3.57 & 0.33 & 2.14 & 0.36 & 0.67 & 0.03 & 0.11 & 0.06 & 188.64 & 181.37 & 15.20 & 11.93 & 0.73 & 7.20 & 7.07 \\
\hline 15XY-29-1-1 & Sch 3 & 91.01 & 143.56 & 14.00 & 50.63 & 6.56 & 1.42 & 5.93 & 0.70 & 3.88 & 0.72 & 1.40 & 0.14 & 0.30 & 0.07 & 320.32 & 307.18 & 32.20 & 9.54 & 0.68 & 8.72 & 11.59 \\
\hline 15XY-29-1-2 & Sch 3 & 56.70 & 111.07 & 14.19 & 67.33 & 13.04 & 2.74 & 13.83 & 1.69 & 9.11 & 1.69 & 3.26 & 0.29 & 0.76 & 0.09 & 295.77 & 265.06 & 77.80 & 3.41 & 0.62 & 2.73 & 19.45 \\
\hline $15 X Y-29-1-3$ & Sch 3 & 54.73 & 107.86 & 15.22 & 83.18 & 16.32 & 3.04 & 20.02 & 2.42 & 12.67 & 2.53 & 4.83 & 0.37 & 1.46 & 0.10 & 324.74 & 280.35 & 112.81 & 2.49 & 0.51 & 2.11 & 25.06 \\
\hline 15XY-29-1-4 & Sch 3 & 81.78 & 129.44 & 14.31 & 59.54 & 11.14 & 2.33 & 13.85 & 1.69 & 8.88 & 1.40 & 3.42 & 0.21 & 0.45 & 0.08 & 328.54 & 298.55 & 72.99 & & & 4.62 & 23.25 \\
\hline $15 X Y-29-1-5$ & Sch 3 & 111.35 & 171.18 & 18.03 & 73.92 & 13.18 & 2.53 & 14.90 & 1.90 & 9.55 & 2.05 & 4.24 & 0.43 & 1.29 & 0.10 & 424.66 & 390.20 & 91.28 & 4.27 & 0.55 & 5.31 & 19.66 \\
\hline $15 X Y-29-1-6$ & Sch 3 & 112.31 & 174.05 & 18.20 & 71.14 & 15.46 & 2.98 & 16.85 & 2.07 & 11.66 & 2.46 & 5.16 & 0.45 & 1.66 & 0.16 & 434.59 & 394.13 & 109.08 & 3.61 & 0.56 & 4.57 & 13.72 \\
\hline 15XY-29-1-7 & Sch 3 & 89.02 & 137.17 & 14.28 & 66.28 & 12.42 & 2.87 & 15.86 & 1.76 & 9.81 & 1.91 & 4.64 & 0.36 & 1.13 & 0.15 & 357.66 & 322.03 & 94. & & 0.63 & 4.51 & 13.63 \\
\hline $15 X Y-29-1-8$ & Sch 3 & 148.15 & 200.34 & 16.91 & 53.49 & 7.52 & 1.45 & 6.07 & 0.81 & 3.96 & 0.82 & 1.76 & 0.15 & 0.41 & 0.09 & 441.93 & 427.86 & & 10.97 & 0.64 & 12.39 & 8.77 \\
\hline $15 X Y-29-1-9$ & Sch 3 & 130.06 & 180.17 & 13.94 & 34.61 & 3.82 & 1.55 & 2.02 & 0.29 & 1.34 & 0.24 & 0.56 & 0.01 & 0.27 & 0.06 & 368.94 & 364.16 & 12. & 28.42 & 1.54 & 21.39 & 4.53 \\
\hline $15 X Y-29-1-10$ & Sch 3 & 100.53 & 139.62 & 11.24 & 27.20 & $\begin{array}{l}.02 \\
1.96\end{array}$ & 0.80 & $\begin{array}{l}2.02 \\
1.69\end{array}$ & 0.12 & 0.61 & 0.10 & 0.26 & 0.03 & 0.02 & 0.07 & 284.25 & 281.35 & 5.93 & 47.44 & 1.32 & 32.25 & 2.96 \\
\hline 15XY-29-1-11 & Sch 3 & 95.33 & 134.30 & 10.26 & 22.99 & 2.44 & 0.81 & 1.85 & 0.26 & 0.95 & 0.19 & 0.31 & 0.01 & 0.18 & 0.06 & 269.94 & 266.14 & 10.17 & 26.18 & 1.12 & 24.57 & 4.14 \\
\hline $15 X Y-31-7$ & Garnet & 14.88 & 35.28 & 4.30 & 17.30 & 3.55 & 0.69 & 3.06 & 0.40 & 2.98 & 0.50 & 1.67 & 0.20 & 0.90 & 0.18 & 85.89 & 76.00 & 25.63 & 2.97 & 0.57 & 5.67 & 0.74 \\
\hline $15 X Y-31-8$ & Garnet & 14.11 & 35.75 & 4.24 & 14.64 & 2.70 & 0.61 & 4.11 & 0.68 & 5.03 & 1.15 & 3.40 & 0.50 & 3.52 & 0.61 & 91.04 & 72.05 & 52.15 & 1.38 & 0.62 & 2.64 & 2.14 \\
\hline 15XY-14-4 & Pyroxene & 0.74 & 1.82 & 0.30 & 1.16 & 0.33 & 0.15 & 0.00 & 0.12 & 0.83 & 0.12 & 0.28 & 0.08 & 1.74 & 0.59 & 8.24 & 4.49 & 8.66 & 0.52 & 0.56 & 3.29 & 0.87 \\
\hline 15XY-14-5 & Pyroxene & 0.28 & 1.35 & 0.22 & 1.67 & 0.45 & 0.17 & 0.14 & 0.09 & 0.63 & 0.14 & 0.43 & 0.18 & 1.87 & 0.53 & 8.15 & 4.15 & 8.95 & 0.46 & 2.41 & 1.40 & 0.00 \\
\hline
\end{tabular}


Table 3. Cont

\begin{tabular}{|c|c|c|c|c|c|c|c|c|c|c|c|c|c|c|c|}
\hline Sample & Sch Type & $\mathrm{Na}$ & Mo & As & $\mathrm{Sr}$ & $Y$ & $\mathrm{Zr}$ & $\mathrm{Nb}$ & $\mathrm{Hf}$ & Ta & $\mathrm{Cd}$ & $\mathrm{Pb}$ & $\mathrm{Zr} / \mathrm{Hf}$ & $\mathrm{Nb} / \mathrm{Ta}$ & $\mathrm{Y} / \mathrm{Ho}$ \\
\hline 15XY-31-1 & Sch 1a & 26.75 & $45,593.04$ & 6.17 & 67.04 & 2.05 & 9.58 & 15.00 & 1.38 & 850.67 & 5.98 & 1.89 & 6.92 & 0.02 & 20.51 \\
\hline $15 X Y-31-3$ & Sch 1a & 12.40 & $20,578.05$ & 3.32 & 82.70 & 2.72 & 9.60 & 4.55 & 1.60 & 863.90 & 2.52 & 0.65 & 6.01 & 0.01 & 20.58 \\
\hline $15 X Y-31-6$ & Sch 1a & 42.02 & $60,560.35$ & 11.00 & 87.15 & 3.49 & 8.87 & 8.96 & 1.61 & 779.38 & 8.30 & 3.05 & 5.52 & 0.01 & 17.86 \\
\hline $15 X Y-31-2$ & Sch $2 a$ & 2.67 & $10,628.82$ & 0.00 & 89.71 & 0.45 & 9.62 & 1.33 & 1.54 & 928.03 & 1.11 & 0.38 & 6.23 & 0.00 & 13.78 \\
\hline $15 X Y-31-4$ & Sch $2 a$ & 0.00 & 8390.98 & 1.43 & 87.84 & 0.60 & 10.24 & 3.34 & 1.63 & 907.67 & 1.02 & 0.67 & 6.29 & 0.00 & 15.74 \\
\hline $15 X Y-31-5$ & Sch 2a & 0.00 & $13,343.18$ & 7.89 & 73.23 & 1.88 & 33.36 & 32.09 & 3.09 & 860.23 & 1.51 & 2.12 & 10.78 & 0.04 & 20.43 \\
\hline 15XY-14-1 & Sch $1 \mathrm{~b}$ & 233.91 & 1083.30 & 12.94 & 102.80 & 245.88 & 11.20 & 10.86 & 1.75 & 1469.78 & 0.34 & 2.90 & 6.41 & 0.01 & 24.55 \\
\hline $15 X Y-14-2$ & Sch $1 \mathrm{~b}$ & 0.00 & 58.80 & 4.29 & 76.72 & 496.78 & 10.98 & 22.19 & 2.19 & 1568.93 & 0.00 & 1.67 & 5.00 & 0.01 & 26.54 \\
\hline 15XY-14-3 & Sch $1 \mathrm{~b}$ & 0.00 & 1386.64 & 12.15 & 110.21 & 154.92 & 13.88 & 10.54 & 2.08 & 1538.07 & 0.00 & 2.60 & 6.68 & 0.01 & 24.42 \\
\hline $15 X Y-14-8$ & Sch $1 \mathrm{~b}$ & 0.00 & 2205.34 & 10.28 & 110.18 & 143.31 & 10.41 & 10.02 & 1.68 & 1397.17 & 0.40 & 3.05 & 6.21 & 0.01 & 23.62 \\
\hline $15 X Y-14-11$ & Sch $1 b$ & 0.00 & 622.94 & 8.58 & 108.78 & 145.40 & 10.03 & 10.18 & 2.17 & 1399.36 & 0.00 & 2.18 & 4.63 & 0.01 & 25.83 \\
\hline 15XY-14-6 & Sch $2 b$ & 0.00 & 5814.94 & 6.17 & 117.73 & 144.85 & 10.86 & 12.09 & 1.73 & 1359.43 & 0.00 & 2.87 & 6.26 & 0.01 & 24.14 \\
\hline $15 X Y-14-7$ & Sch $2 b$ & 256.49 & $86,771.32$ & 10.90 & 159.58 & 105.49 & 35.85 & 12.88 & 1.72 & 1124.92 & 7.42 & 3.76 & 20.83 & 0.01 & 34.50 \\
\hline $15 X Y-14-9$ & Sch $2 b$ & 0.00 & $47,068.48$ & 11.83 & 144.46 & 195.47 & 36.36 & 46.83 & 2.84 & 1181.12 & 1.98 & 4.34 & 12.80 & 0.04 & 32.14 \\
\hline $15 X Y-14-10$ & Sch $2 b$ & 28.53 & $56,974.31$ & 18.48 & 154.41 & 118.61 & 13.98 & 26.22 & 1.81 & 1218.51 & 4.56 & 5.07 & 7.72 & 0.02 & 30.28 \\
\hline 15XY-14-12 & Sch $2 b$ & 0.00 & $53,754.93$ & 13.20 & 141.41 & 193.92 & 44.58 & 53.07 & 3.05 & 1125.68 & 4.04 & 4.11 & 14.63 & 0.05 & 32.52 \\
\hline $15 X Y-29-2-1$ & Sch 3 & 179.15 & $44,234.77$ & 14.68 & 104.95 & 3.35 & 12.42 & 16.21 & 1.56 & 1088.53 & 5.39 & 27.70 & 7.96 & 0.01 & 31.80 \\
\hline $15 X Y-29-2-2$ & $\operatorname{Sch} 3$ & 78.46 & $52,207.24$ & 2.31 & 113.54 & 11.64 & 11.88 & 6.58 & 1.65 & 1065.89 & 3.46 & 2.70 & 7.18 & 0.01 & 24.77 \\
\hline $15 X Y-29-2-3$ & Sch 3 & 111.19 & $56,637.38$ & 5.12 & 110.60 & 8.79 & 10.62 & 13.94 & 1.62 & 1068.39 & 52.76 & 15.05 & 6.56 & 0.01 & 33.58 \\
\hline $15 X Y-29-2-4$ & Sch 3 & 65.81 & $61,604.73$ & 9.68 & 103.10 & 5.29 & 10.51 & 8.02 & 1.53 & 1063.14 & 6.19 & 2.26 & 6.85 & 0.01 & 30.03 \\
\hline $15 X Y-29-2-5$ & $\operatorname{Sch} 3$ & 68.74 & $72,813.94$ & 4.49 & 93.14 & 5.62 & 9.74 & 5.24 & 1.25 & 1048.24 & 6.56 & 2.09 & 7.78 & 0.00 & 43.82 \\
\hline $15 X Y-29-2-6$ & Sch 3 & 200.18 & 89824.21 & 5.79 & 106.47 & 14.85 & 10.63 & 4.27 & 1.35 & 990.88 & 6.87 & 1.76 & 7.86 & 0.00 & 33.23 \\
\hline $15 X Y-29-2-7$ & Sch 3 & 0.00 & $11,675.75$ & 1.32 & 109.74 & 74.48 & 10.41 & 25.06 & 1.63 & 1170.84 & 0.55 & 1.13 & 6.39 & 0.02 & 29.59 \\
\hline $15 X Y-29-2-8$ & Sch 3 & 147.39 & $75,612.31$ & 8.22 & 114.14 & 142.69 & 11.99 & 11.76 & 1.32 & 1048.01 & 4.92 & 1.90 & 9.09 & 0.01 & 32.27 \\
\hline $15 X Y-29-2-9$ & Sch 4 & 44.97 & $32,894.58$ & 2.07 & 89.23 & 16.35 & 9.18 & 26.99 & 1.50 & 1179.63 & 6.15 & 1.99 & 6.11 & 0.02 & 26.46 \\
\hline $15 X Y-29-2-10$ & Sch 4 & 0.00 & 17636.97 & 1.75 & 87.58 & 7.93 & 10.86 & 21.35 & 1.57 & 1186.75 & 1.09 & 5.71 & 6.93 & 0.02 & 22.32 \\
\hline $15 X Y-29-1-1$ & $\operatorname{Sch} 3$ & 0.00 & 86685.10 & 18.00 & 119.72 & 19.05 & 10.18 & 7.23 & 1.32 & 1055.65 & 1.81 & 2.91 & 7.70 & 0.01 & 26.51 \\
\hline $15 X Y-29-1-2$ & Sch 3 & 0.00 & 24606.24 & 5.14 & 102.35 & 47.09 & 9.67 & 23.11 & 1.74 & 1268.90 & 1.16 & 1.79 & 5.56 & 0.02 & 27.92 \\
\hline $15 X Y-29-1-3$ & Sch 3 & 75.71 & $18,813.40$ & 2.73 & 101.12 & 68.41 & 10.52 & 29.70 & 1.54 & 1295.13 & 4.77 & 1.59 & 6.84 & 0.02 & 27.07 \\
\hline $15 X Y-29-1-4$ & $\operatorname{Sch} 3$ & 16.59 & $17,920.21$ & 4.70 & 92.02 & 42.99 & 9.85 & 23.06 & 1.30 & 1238.47 & 8.31 & 3.08 & 7.59 & 0.02 & 30.67 \\
\hline $15 X Y-29-1-5$ & $\operatorname{Sch} 3$ & 59.33 & $69,605.23$ & 4.98 & 101.20 & 56.82 & 13.04 & 11.56 & 1.26 & 1078.53 & 1.32 & 7.00 & 10.33 & 0.01 & 27.76 \\
\hline $15 X Y-29-1-6$ & Sch 3 & 82.39 & $79,410.89$ & 9.31 & 104.63 & 68.61 & 12.12 & 9.30 & 1.42 & 1090.63 & 10.03 & 8.46 & 8.53 & 0.01 & 27.87 \\
\hline $15 X Y-29-1-7$ & $\operatorname{Sch} 3$ & 0.00 & $16,264.14$ & 5.37 & 102.81 & 58.83 & 10.10 & 24.79 & 1.88 & 1242.52 & 2.90 & 1.76 & 5.37 & 0.02 & 30.79 \\
\hline $15 X Y-29-1-8$ & $\operatorname{Sch} 3$ & 0.00 & $89,459.70$ & 4.95 & 106.96 & 24.91 & 11.35 & 3.96 & 1.44 & 963.19 & 4.50 & 2.25 & 7.87 & 0.00 & 30.27 \\
\hline $15 X Y-29-1-9$ & Sch 3 & 63.93 & $59,743.58$ & 10.98 & 106.49 & 8.03 & 11.28 & 9.49 & 1.89 & 1102.08 & 6.28 & 2.58 & 5.96 & 0.01 & 33.40 \\
\hline $15 X Y-29-1-10$ & $\operatorname{Sch} 3$ & 0.00 & $51,315.64$ & 14.24 & 120.36 & 3.03 & 10.39 & 8.60 & 1.73 & 1124.43 & 1.52 & 1.80 & 5.99 & 0.01 & 29.80 \\
\hline $15 X Y-29-1-11$ & Sch 3 & 70.21 & $91,626.28$ & 9.87 & 112.34 & 6.36 & 10.81 & 6.68 & 1.53 & 984.72 & 2.59 & 2.05 & 7.08 & 0.01 & 34.15 \\
\hline Sample & Sch Type & $\mathrm{Na}$ & Mo & As & $\mathrm{Sr}$ & $Y$ & $\mathrm{Zr}$ & $\mathrm{Nb}$ & $\mathrm{Hf}$ & $\mathrm{Ta}$ & $\mathrm{Au}$ & $\mathrm{Pb}$ & $\mathrm{Zr} / \mathrm{Hf}$ & $\mathrm{Nb} / \mathrm{Ta}$ & $\mathrm{Y} / \mathrm{Ho}$ \\
\hline $15 X Y-31-7$ & Garnet & 384.43 & 7.38 & 1.14 & 0.24 & 15.74 & 7.04 & 1.68 & 0.17 & 0.55 & 0.01 & 0.47 & 41.89 & 3.07 & 31.31 \\
\hline $15 X Y-31-8$ & Garnet & 219.27 & 4.22 & 2.68 & 0.49 & 33.16 & 37.89 & 5.51 & 1.61 & 0.64 & 0.00 & 0.26 & 23.60 & 8.60 & 28.95 \\
\hline $15 X Y-14-4$ & Pyroxene & 0.06 & 0.00 & 1.81 & 11.67 & 4.91 & 6.41 & 0.06 & 0.00 & 0.01 & 0.00 & 0.24 & 0.00 & 10.87 & 39.66 \\
\hline $15 X Y-14-5$ & Pyroxene & 0.18 & 0.23 & 0.00 & 7.41 & 4.95 & 6.03 & 0.00 & 0.50 & 0.00 & 0.02 & 27.47 & 12.03 & 0.00 & 36.03 \\
\hline
\end{tabular}


Sch3 has different REE patterns in a single grain and varying total REE contents of 162.9-890.1 ppm (Figure 7e-h). The change from core to rim in a single Sch3 grain shows a systematic change from negative to positive Eu-anomaly $\left(\mathrm{Eu} / \mathrm{Eu}^{*}=0.51-4.89\right)$ and progressive increase in the LREE/HREE ratios of 2.5-96.2 (Figure 7e-f). Compared with Sch2a, Sch3 have higher Sr contents of 92.0-185.8 ppm and $Y$ contents of 3.0-142.6 ppm.

Sch4 displays the same REE patterns as the core of Sch3 with low LREE/HREE ratios of 8.4-11.9, lower total REE contents of 188.6-249.1 ppm and Eu/Eu* ratios of 0.40-0.73, compared with those of Sch3 (Figure 7g,h). Sch4 has lower Sr contents, 87.6-89.2 ppm, and Y contents, 7.9-16.4 ppm, than those of Sch3.
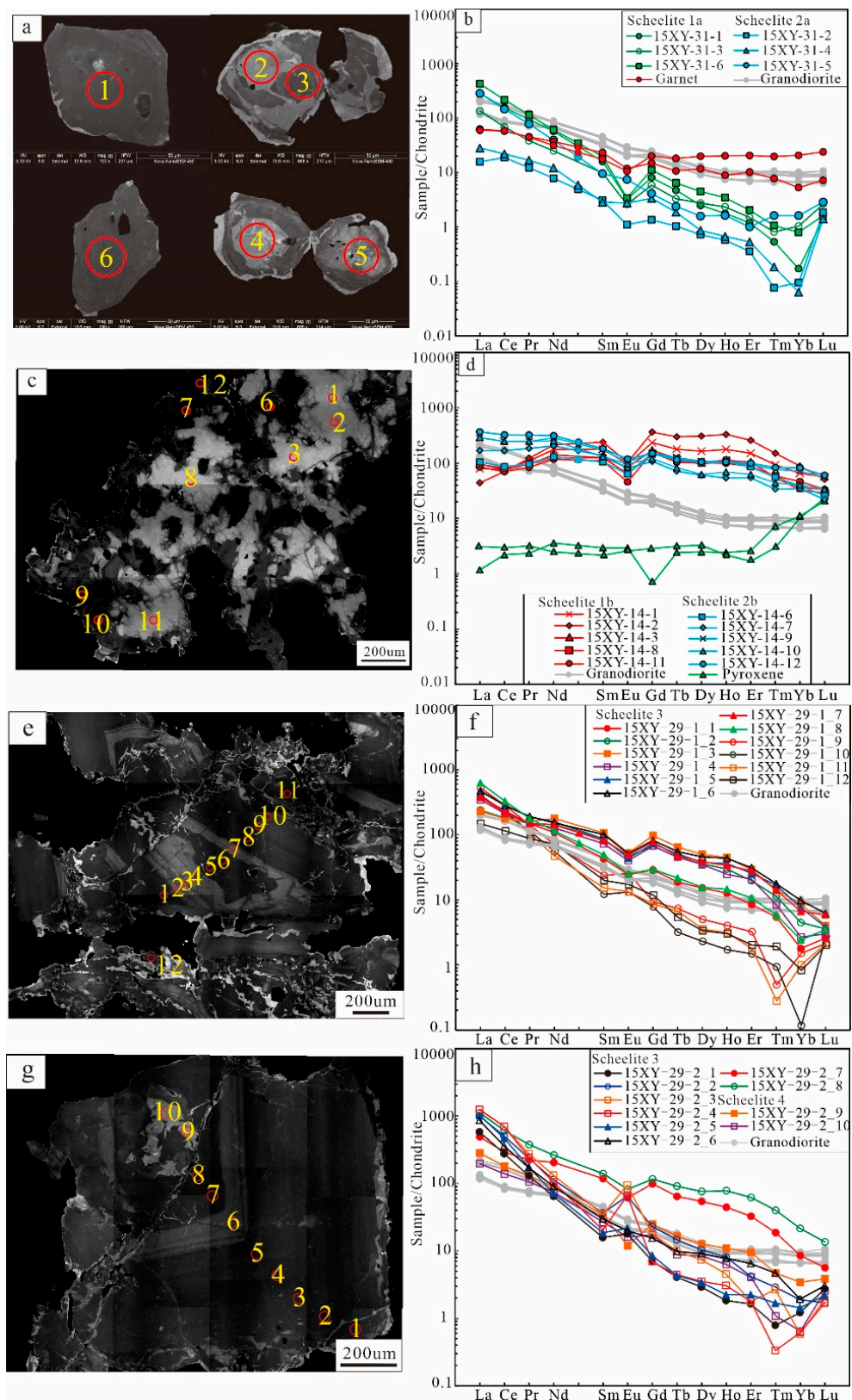

Figure 7. Chondrite-normalized REE patterns of scheelites from Xiaoyao deposit. (a) and (b) are Sch1a and Sch2a in the garnet skarn, sample 15XY-31; (c) and (d) are Sch1b and Sch2b in the pyroxene skarn, sample 15XY-14; (e-h) are Sch3 and Sch4 hosted in scheelite-rich quartz veins, sample 15XY-29. The chondrite values are from [33]. The granodiorite data are from [28]. 


\section{Discussion}

\subsection{Oxidation State of Ore-Forming Fluids}

Molybdenum cation $\mathrm{Mo}^{6+}$ enters scheelite by substituting $\mathrm{W}^{6+}$ under high $\mathrm{fO}_{2}$ conditions; under low $f \mathrm{O}_{2}$ and high $f \mathrm{~S}_{2}$ conditions, $\mathrm{Mo}^{6+}$ is reduced to $\mathrm{Mo}^{4+}$ and precipitates as molybdenite. Thus, the molybdenum contents within scheelite crystals can be used to indicate the oxidation conditions of ore-forming fluids [34-36]. In the proximal zone, the $\mathrm{MoO}_{3}$ contents of Sch1a are high with 5.77-10.30\%, indicating oxidized conditions (Figure 8A). The redox state of early ore-forming fluids could be inherited from magmas, and Xiaoyao granodiorite is oxidized. The existence of accessory magnetite in the Xiaoyao granodiorite indicates that the magmas had a high oxygen fugacity. Sch1b in the distal zone has lower $\mathrm{MoO}_{3}$ contents of $0.38-0.42 \%$ (Table 2 and Figure 8B). The carbonaceous wall rocks may lessen the effects of such a relatively oxidized magma $[17,18]$. However, the lack of association between molybdenite and Sch1b under reduced conditions suggests that the ore solution may be already depleted in molybdenum and/or under low $f S_{2}$ conditions [34]. The activity of molybdenum decreases from the proximal zone to the distal zone [16]. In addition, Sch2b and Sch3 have higher $\mathrm{MoO}_{3}$ contents (8.07-10.17\% and 4.09-9.78\%, respectively), compared with Sch1b and Sch2a (Table 2 and Figure $8 \mathrm{E}-\mathrm{G})$. The late oxidized Mo-rich granodiorite dike overprints the sample location of Sch $2 \mathrm{~b}$ (Figure 3), and Sch2b and Sch3 have the same REE patterns with ore-related granodiorite (Figure 7d,f). The elevated Mo content may be provided by the high oxidation state, as well as an overprinting or remobilization of the oxidized Mo-rich magmatic-hydrothermal system [17]. However, Sch2a and Sch4 have low $\mathrm{MoO}_{3}$ contents (1.71-3.51\% and 0.19-0.76\%, respectively), accompanied by $\mathrm{MoS}_{2}$ precipitation (Figure $8 \mathrm{C}, \mathrm{H}$ ). These variations may be related to low $f \mathrm{O}_{2}$ and high $f \mathrm{~S}_{2}$, caused by fluid-rock interaction. Furthermore, the precipitation of Mo-rich scheelite likely resulted in a Mo-depletion in residual fluids. The varying redox state of ore-forming fluids is influenced by the replenishment of oxidized Mo-rich magmatic-hydrothermal fluids and various interactions with carbonaceous wall rocks.

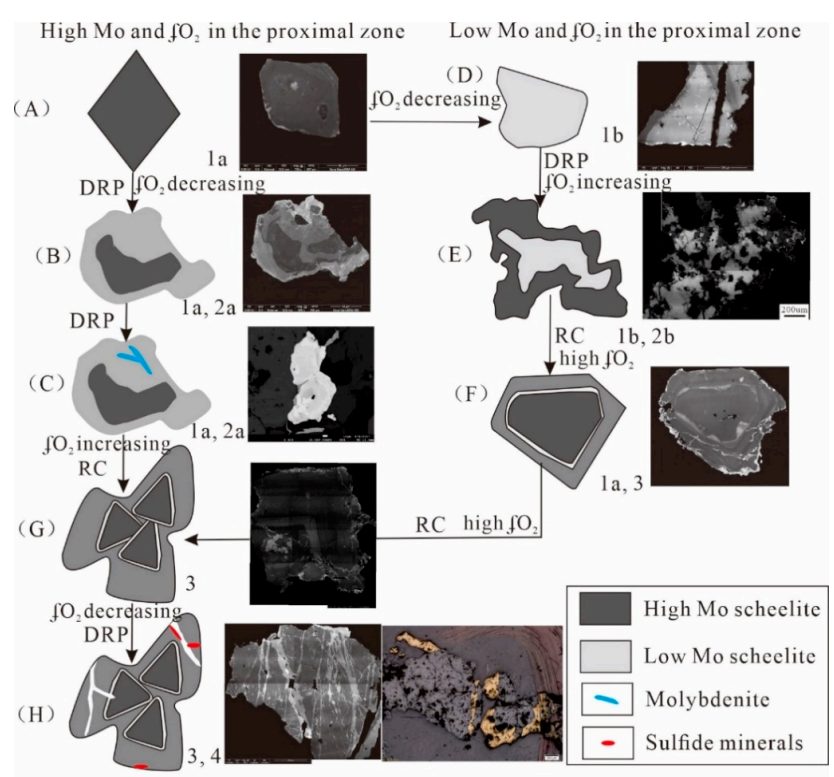

Figure 8. Sketches illustrating multiple re-equilibration processes in scheelite from the Xiaoyao tungsten skarn deposit. The high-Mo scheelite in the proximal zone has undergone the dissolution and re-precipitation, forming some molybdenite grains $(\mathbf{A}, \mathbf{B}, \mathbf{C})$. The low-Mo scheelite grains in the distal zone (D) have extensively re-equilibrated by DRP (E). Scheelite grains from the quartz-scheelite-sulfide stage comprise the homogeneous core and growth-zoning rim (F,G). Both high-Mo and low-Mo scheelites may have been subjected to recrystallization $(\mathbf{F}, \mathbf{G})$ and then locally modified by DRP $(\mathbf{H})$. For more details, see the text. Abbreviations: DRP $=$ dissolution and re-precipitation, $\mathrm{RC}=$ re-crystallization. The numbers 1 to 4 refer to the types of scheelite. 
The presented results indicate a positive correlation between Mo and Cd in the scheelite (Figure 9a); therefore, the $\mathrm{Cd}$ content in scheelite increases under oxidized conditions. In contrast, under reduced conditions, the $\mathrm{Cd}$ content of scheelite decreases. This is corroborated by the changing valence state of $\mathrm{Cd}$ in an evolving ore forming fluid. $\mathrm{Cd}$ has two valence states, i.e. +1 and +2 . $\mathrm{Cd}$ migrates as $\mathrm{Cd}^{1+}$ and its ionic radius is $1.14 \AA$; Cd migrates as $\mathrm{Cd}^{2+}$ and its ionic radius is $0.97 \AA$ [37]. $\mathrm{Cd}^{2+}$ has the same valence states as $\mathrm{Ca}^{2+}$ and its ionic radius is close to $0.99 \AA$ of $\mathrm{Ca}^{2+}$ and preferentially enters scheelite by substituting $\mathrm{Ca}^{2+}$ in fluids under oxidized conditions. The $\mathrm{Cd}$ average content of scheelite in the proximal skarn is $5.60 \mathrm{ppm}$, while, in the distal skarn, it is 0.15 ppm (Table 3), indicating lower oxidized conditions in the distal zone. These results are consistent with the lower Mo contents of the distal zone. This suggests that, in addition to the Mo content, the Cd content of scheelite can also be used to estimate the redox conditions of ore-forming fluids.
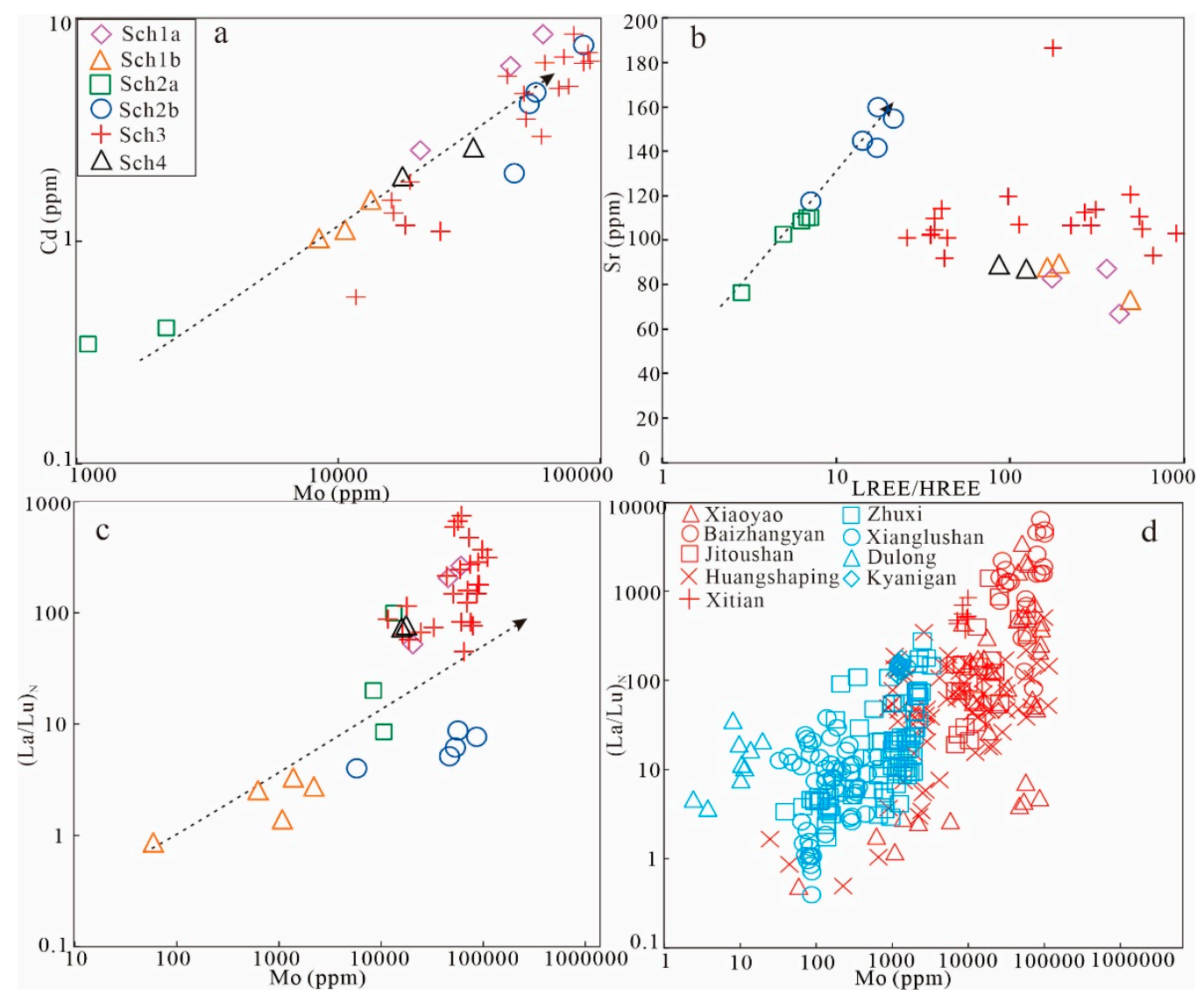

Figure 9. (a) Plot of Mo vs. Cd. (b) Plot of LREE/HREE vs. Sr. (c,d) Plot of Mo vs. $(\mathrm{La} / \mathrm{Lu})_{\mathrm{N}}$. the scheelite data from the oxidized tungsten skarn deposits, including the Jitoushan and Baizhangya [9], Xitian [38]; the reduced tungsten skarn deposits, including the Zhuxi [15], Xianglushan [14], Kyanigan [39].

\subsection{Disequilibrium Processes in Scheelite.}

\subsubsection{Dissolution and Re-Precipitation}

The majority of the analyzed skarn-related scheelite has been extensively modified by dissolution and re-precipitation (DRP) processes. The high-Mo primary Sch1a is replaced to various extents by a secondary variety along fractures and/or grain boundaries (Figure 8A,B). This high-Mo Sch1a may have originally formed at high temperatures and under relatively oxidizing conditions. Furthermore, it was subsequently modified through the decrease in $\mathrm{MoO}_{3}$ contents and molybdenite precipitation, caused by a partial reduction of $\mathrm{Mo}^{6+}$ to $\mathrm{Mo}^{4+}$ in response to decreasing $f \mathrm{O}_{2}$. Secondary Sch2a is generally in sharp contact with the primary Sch1a and intergrown with molybdenite (Figure 8C). Low-Mo Sch1b 
has been modified by DRP processes (Figure 8D,E) through increases om $\mathrm{MoO}_{3}$ contents, caused by the substitution of $\mathrm{W}^{6+}$ and $\mathrm{Mo}^{6+}$ in response to increasing $f \mathrm{O}_{2}$. The replacement of garnet released large amounts of calcium ion into the ore-forming fluid and thus raised the activity of calcium ion in solution. Consequently, this led to a disequilibrium between precipitated scheelite and evolving fluids $[16,17]$. In addition, the $\mathrm{W}$ contents of garnet from the Xiaoyao deposit are 219-384 ppm (Table 3); therefore, garnet breakdown can lead to disequilibrium. Fluid-rock interaction led to the dissolution of the primary scheelite and the formation of secondary varieties [35]. These have been identified as important components in the formation of Xiaoyao tungsten skarn and similar deposits in the same district. Carbonate rocks contributed to an increase in the Ca contents of ore-forming fluids, enhanced $\mathrm{WO}_{4}{ }^{2-}$ solubility, and consequently led to disequilibrium. In addition, the re-equilibration of scheelite must have resulted in an increase in the extent of grain boundaries. This is favorable for fluid infiltration and further promotes the dissolution and re-precipitation of scheelite.

\subsubsection{Recrystallization}

Sch3 displays a homogeneous core and growth zoning rim (Figure 8F,G). In this study, scheelite grains with distinctive texture comprise a Mo-rich core, and a low-Mo rim (Figure 8F,G). Both generations have significantly different trace element compositions (Figure 7e-h). Both the oxidation state and the types of REE complexing may also be important during the recrystallization of scheelite and may have caused the observed LREE/HREE fractionation. Sch3 grains were modified by DRP processes and were replaced to various extents by Sch4 along fractures together with $\mathrm{MoS}_{2}$ and other sulfide precipitations (e.g., chalcopyrite and pyrrhotite) (Figure $8 \mathrm{H}$ ). Therefore, scheelite from the Xiaoyao tungsten skarn deposit can be concluded to have undergone complex re-equilibration processes, where the precursor scheelite was partially modified or locally completely destroyed. Identification of such multiple re-equilibration processes reflects the dynamic changes in the fluid systems that are responsible for tungsten skarn formation.

\subsection{Control of Chemical Variation in Scheelite.}

Scheelite contains multiple groups of trace elements, such as REEs, LILEs ( $\mathrm{Sr}$ and $\mathrm{Pb}$ ), Mo, As, and HFSEs ( $\mathrm{Y}, \mathrm{Nb}$, and $\mathrm{Ta}$ ) (Table 3). Scheelite can incorporate significant amounts of REEs, $\mathrm{Sr}, \mathrm{Pb}$, and $\mathrm{Y}$ because of substitution for $\mathrm{Ca}^{2+}$, while $\mathrm{Mo}, \mathrm{As}, \mathrm{Nb}$ and $\mathrm{Ta}$ enter scheelite via substitution for $\mathrm{W}^{6+}[5]$. Subtle variations in the trace element geochemistry of scheelite samples from this study were found between sample locations and scheelite types; the most significant include variations in $\mathrm{Mo}, \mathrm{Sr}$, Y, LREEs, and HREEs.

Different substitution mechanisms lead to different chondrite-normalized REE patterns of scheelites [5]. Three main mechanisms have been identified as responsible for REE substitution [5]: (1) $2 \mathrm{Ca}^{2+}=\mathrm{REE}^{3+}+\mathrm{Na}^{+}$; (2) $\mathrm{Ca}^{2+}+\mathrm{W}^{6+}=\mathrm{REE}^{3+}+\mathrm{Nb}^{5+}$; and (3) $3 \mathrm{Ca}^{2+}=2 \mathrm{REE}^{3+}+\mathrm{Ca}$, where Ca is a Ca-site vacancy. Regarding mechanism (1), MREEs preferentially substitute into a Ca site because of their similar ionic radii if $\mathrm{Na}$ is available to provide the charge balance in scheelite. Mechanism (2) requires that scheelites are $\mathrm{Nb}^{5+}$-enriched and have $\mathrm{Nb}$ contents close to the $\Sigma \mathrm{REEs}$, with a positive correlation of $\mathrm{Nb}^{5+}$ and $\mathrm{Nd}^{3+}$ [6]. As shown in Figure 7, none of the scheelites from Xiaoyao display MREE-enriched REE patterns, and contain very low Nb contents of 1.33-38.45 ppm, which are much lower than their REE contents of 21-1032 ppm (Table 3). This indicates that mechanism (3) is more applicable than either (1) or (2). Because of the vacancy in mechanism (3), the restrictions of ionic radii were slightly modified, and the scheelite may inherit its REE composition from ore-forming fluids [9]. Sch1a, Sch2a, Sch3, and Sch4 in the proximal zone display LREE-enriched and slightly HREE-depleted patterns (Figure 7). These are similar to the pattern of ore-related granodiorite, indicating that the scheelites inherited REE compositions from magmatic-hydrothermal fluids. However, they have higher LREE/HREE ratios compared with the ore-related granodiorite which may be caused by the precipitation of skarn minerals with low LREE/HREE ratios of 1.4-3.0 (Figure 7b). Many researchers reported that the REE patterns of skarn-type scheelites are enriched in LREEs but depleted in HREEs, 
which is likely caused by the precipitation of HREE-rich skarn minerals [9]. Prominent examples include the Zhuxi and Jitoushan tungsten skarn deposits in the JNB [9] and the Kyanigan tungsten skarn deposit in the Mogok metamorphic belt, Myanmar [39]. However, the Sch1b and Sch2b of the distal zone with low LREE/HREE ratios of 0.3-2.1 were identified by their distinctive REE fractionation patterns (Figure 7d). Such low LREE/HREE ratios can be interpreted by several explanations. Firstly, mineral/mineral REE partitioning may have occurred. However, because pyroxene intergrowth with scheelite has much lower REE contents of 0.6-13.1 ppm (Table 3), this is insufficient to change the LREE/HREE ratios, and thus REE partitioning cannot be explained via this process. Secondly, if the HREE-enriched patterns were assumed to be inherited from the ore-forming fluids, the ore-forming fluids should be HREE-enriched; in contrast to the LREE-enriched patterns of ore-related granodiorite, the inherited process cannot be explained. Thirdly, a minor distortion in $\mathrm{Ca}^{2+}$ will result in a shift from LREE enrichment to HREE enrichment, depending on the reduction in the relative size of the crystallographic site from light to heavy REE (i.e., from $1.12 \mathrm{~A}^{\circ}$ to $1.00 \mathrm{~A}^{\circ}$ ) [40]. The extensive replacement of $\mathrm{Ca}^{2+}$ for $\mathrm{Sr}^{2+}$ may be linked to site distortions because of differences in their ionic radii $\left(\mathrm{Ca}^{2+}, 1.12 \mathrm{~A}^{\circ} ; \mathrm{Sr}^{2+}, 1.26 \mathrm{~A}^{\circ}\right)$ [40]. Sch1b and Sch2b in the distal zone are characterized by low LREE/HREE ratios and relatively low $\mathrm{Sr}$ contents of 76.7-159.6 ppm. Both display a positive correlation between LREE/HREE ratios and Sr contents (Figure 8b), which may be slightly influenced by $\mathrm{Ca}^{2+}$ site distortions.

Moreover, changes from LREE- to HREE-enrichment correlate with variation in $\mathrm{MoO}_{3}$ contents in the distal zone (Figure 9c). This is corroborated by high $\mathrm{MoO}_{3}$ contents of $8.06-10.17 \%$ for Sch $2 b$ and low $\mathrm{MoO}_{3}$ contents of $0.38-0.42 \%$ for Sch1b in the distal zone (Table 2 and Figure 6a), suggesting that scheelites evolved from LREE-enrichment to HREE-enrichment because of a shift in $f \mathrm{O}_{2}$. This is consistent with the change from HREE-enrichment to LREE-enrichment because of a shift in $f \mathrm{O}_{2}$ [41]. Figure $9 \mathrm{a}, \mathrm{b}$ shows positive correlations between $\mathrm{Mo}$ and $(\mathrm{La} / \mathrm{Lu})_{\mathrm{N}}$ in scheelite from Xiaoyao and in other tungsten skarn deposits. Furthermore, the positive correlations between Mo and LREEs ( $\mathrm{La}, \mathrm{Ce}$, and Nd) (Figure 10a-c) gradually change to negative corrections between Mo and HREEs (Gd, Dy, and Ho) (Figure 10d-f). This indicates that the redox state may directly or indirectly influence the REE patterns of Sch1b and Sch2b.

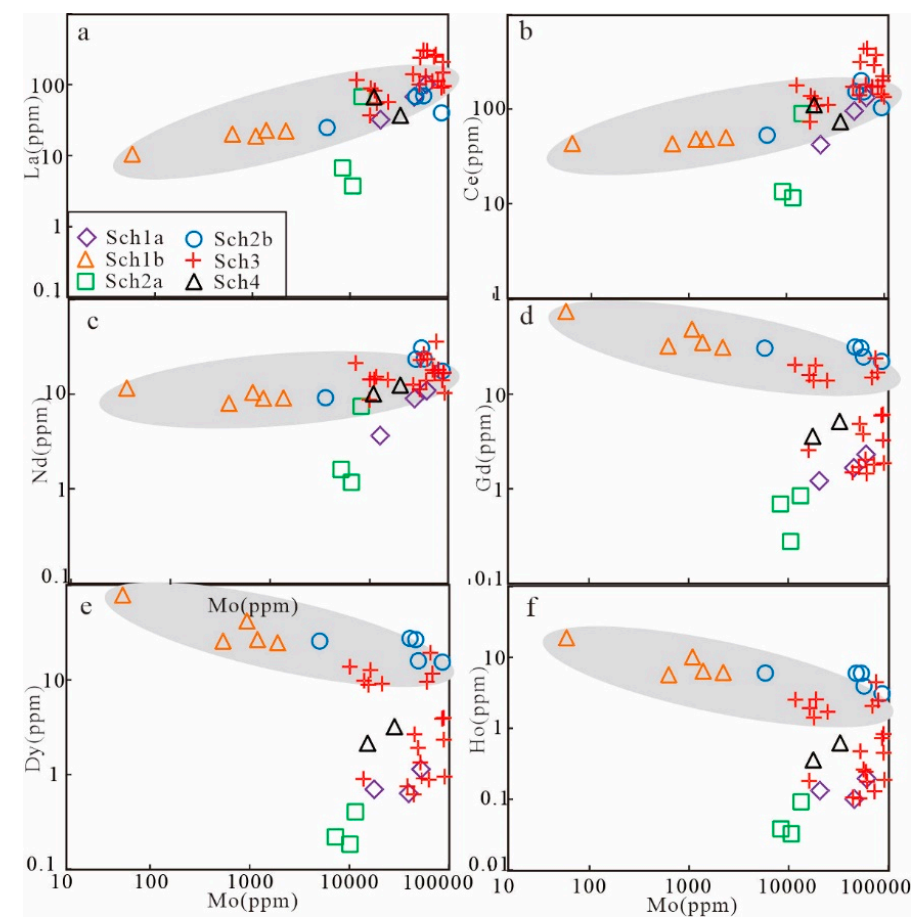

Figure 10. Chemical Variation in Scheelite: (a) Plot of Mo vs. La. (b) Plot of Mo vs. Ce. (c) Plot of Mo vs. Nd. (d) Plot of Mo vs. Gd. (e) Plot of Mo vs. Dy. (f) Plot of Mo vs. Ho. 
Strontium concentrations of Sch1b in the distal zone (76-110 ppm) exceed those of Sch1a in the proximal zone (67-87 ppm; Figure 11e). Furthermore, the Sr contents of Sch2a, Sch2b, and Sch3 are higher compared with those of Sch1a and Sch1b (Figure 11e). This may be attributed to fluid-rock interactions, which, in general, elevate $\mathrm{Sr}$ contents due to Ca-bearing minerals such as carbonates (i.e., as a substitution for $\mathrm{Ca}^{2+}$ ) during wall-rock alteration by the tungsten-bearing fluid $[42,43]$. Many studies of tungsten deposits reported fluid-rock interactions as important processes during mineralization, especially in skarn deposits [44]. Studies of the Sr content and isotope on scheelite from the Felbertal tungsten deposit, Austria [45], and the Dahutang tungsten deposit [33] indicated that fluid-rock interaction and alterations in host rocks by granite-derived hydrothermal fluids can cause the observed Sr enrichment. The lower Sr concentrations of Sch4 in the quartz vein (Figure 11e) may be due to dilution via mixing of magmatic fluids with externally-derived (meteoric) fluids [40], which is consistent with the lower REE contents. The large variation in Sr contents in scheelite reflect the changing Sr composition of the mineralizing fluids, which is, in turn, influenced by fluid-rock interaction and fluid mixing.

Yttrium concentrations in scheelite also vary substantially (0.5-496.8 ppm; Figure 11a-d). Yttrium is positively correlated with REEs, LREEs, and especially HREEs (Figure $11 \mathrm{~b}-\mathrm{d}$ ), which is likely because $\mathrm{Y}$ has a similar charge to $\mathrm{REE}^{3+}$ and a similar ionic radius in eightfold coordination (1.015 $\AA$ ), near Ho (1.02 A) [5]. Similar to HREE, Y also displays negative correlations with Mo (Figure 11a) and is related to the redox state. However, both Sch1a and Sch2a deviate from this correlation. Garnet intergrowth with Sch1a and Sch2a has significant $Y$ contents (i.e., a Y content of 24.45 ppm; Table 3), suggesting that its precipitation may influence $Y$, which is depleted in Sch1a and Sch2a. Variations in $Y$ concentrations in the Xiaoyao scheelite most likely relate to the redox state or the precipitation of Y-rich hydrothermal minerals.
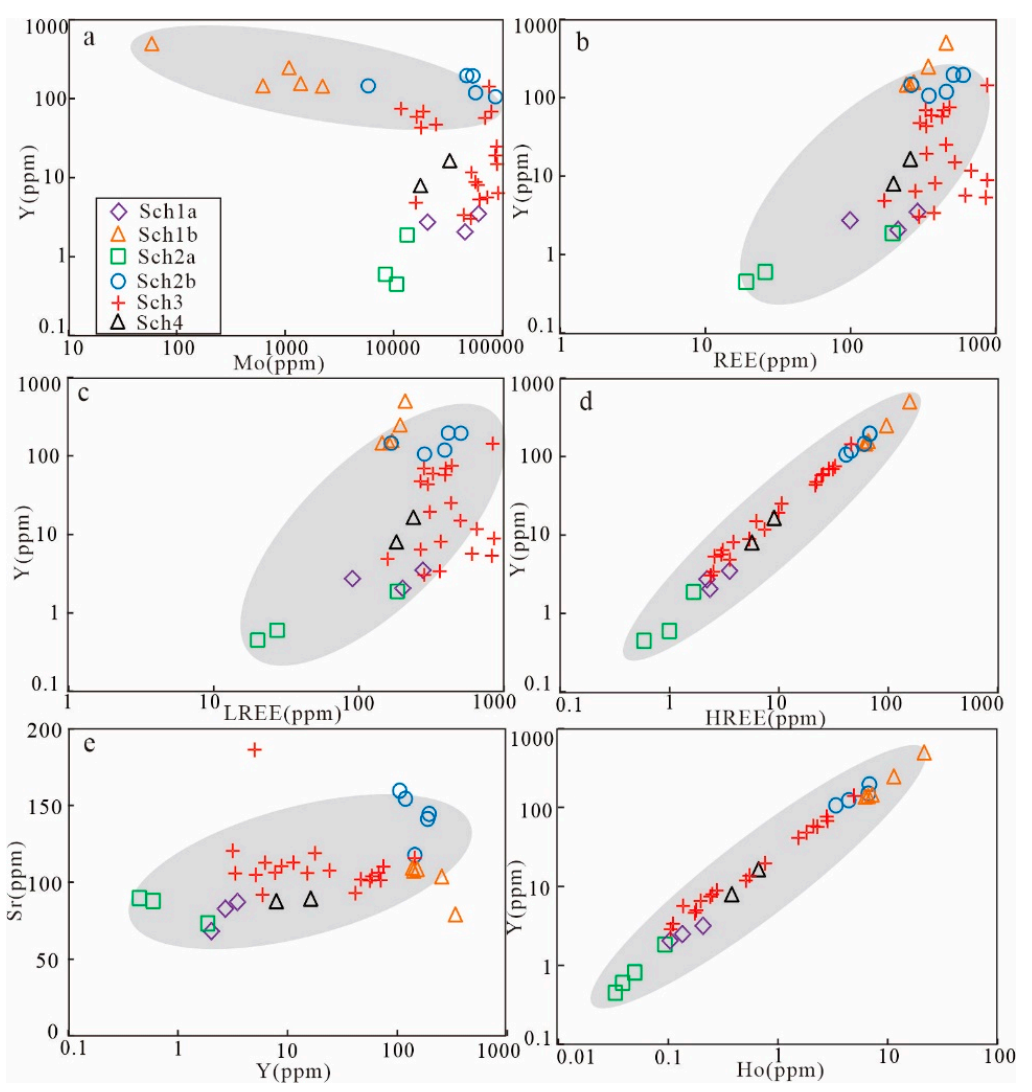

Figure 11. Chemical Variation in Scheelite: (a) Plot of Mo vs. Y. (b) Plot of REE vs. Y. (c) Plot of LREE vs. Y. (d) Plot of HREE vs. Y. (e) Plot of Y vs. Sr. (f) Plot of Ho vs. Y. 
Because of their similar charge and ionic radii, $\mathrm{Y}-\mathrm{Ho}, \mathrm{Zr}-\mathrm{Hf}$, and $\mathrm{Nb}-\mathrm{Ta}$ pairs display coherent behavior and hold their respective chondritic ratios in mafic to intermediate rocks [46]. In the present study, $\mathrm{Y}$ and Ho are positively correlated with $\mathrm{Y} / \mathrm{Ho}$ of $13.78-43.82$, whereas $\mathrm{Zr}-\mathrm{Hf}$ and $\mathrm{Nb}-\mathrm{Ta}$ relationships displayed widely scattered patterns with $\mathrm{Zr} / \mathrm{Hf}$ of $4.63-20.83$ and $\mathrm{Nb} / \mathrm{Ta}<0.05$ (Table 3). The positive correlation between $\mathrm{Y}$ and Ho suggests their similar chemical behavior in hydrothermal systems [46]. However, an explanation for the deviation of these ratios from the chondritic values is still under debate. These elements are mainly concentrated in minor/accessory minerals, the crystallization of which can modify the ratio [47]. Trace element fractionation by scheelite in the hydrothermal system leads to the variable non-chondritic $\mathrm{Y} / \mathrm{Ho}$ and $\mathrm{Zr} / \mathrm{Hf}$ ratios of scheelite [39]. The $\mathrm{Y} / \mathrm{Ho}$ ratios of scheelite from Xiaoyao gradually increase (17.86-26.54, 13.70-34.50, and 22.32-43.82) from the skarn stage to the quartz vein stage. This may be due to fractionation caused by fluid-rock interaction and metasomatism [48] or the breakdown of skarn minerals with high Y/Ho (28.94-39.65, Table 3). The $\mathrm{Zr} / \mathrm{Hf}$ ratios of scheelite increase (4.63-6.92 and 6.23-20.83) from the skarn stage to the retrograde alteration stage, which may be due to fractionation or the breakdown of skarn minerals with high $\mathrm{Zr} / \mathrm{Hf}$ (average of 19.38, Table 3). The $\mathrm{Zr} / \mathrm{Hf}$ ratios of scheelite decrease (5.37-10.33) in the quartz-vein stage, which may be due to the precipitation of hydrothermal zircon. This suggestion is supported by the mineral assemblage of hydrothermal zircon, which is intergrown with scheelite in the quartz-vein stage (Figure 5 h). Compared with most geological materials (e.g., $\mathrm{Nb} / \mathrm{Ta}$ of chondrites $~ 17.5$; average continental crust $\sim 11)[49]$, scheelites have a very low $\mathrm{Nb} / \mathrm{Ta}$ ratio $(<0.05)$, reflecting relatively high abundances of Ta. Fluid fractionation leads to an enrichment of Ta relative to $\mathrm{Nb}$ [50]. The data in this paper support this model and show that hydrothermal fluids efficiently fractionate the $\mathrm{Nb} / \mathrm{Ta}$ ratio.

Based on the above textural and geochemical characteristics, the REE and trace element characteristics of scheelites are influenced by substitution mechanisms, fluid-rock interaction, redox state, fluid mixing, fluid fractionation, and mineral/mineral REE partitioning. The texture may reflect multiple fluid pluses with fluctuating chemical properties and depositional conditions and may indicate modification of the pre-existing scheelite by late-stage fluids in the ore-system.

\subsection{Implication for Ore-Forming Processes}

Granodiorite has a zircon U-Pb isotope age of $149.4 \pm 1.1 \mathrm{Ma}$, which is consistent with the molybdenite Re-Os age ( $148.7 \pm 2.5 \mathrm{Ma})$ at the Xiaoyao deposit, suggesting that it is genetically related to skarn formation and mineralization [28]. Paragenetic studies on textural and physicochemical data show that the Xiaoyao skarn may be classified as an oxidized tungsten deposit that can form in an evolving magmatic-hydrothermal system. Alterations and mineralizations at the Xiaoyao have been associated with calc-alkaline magmatic activity.

The tungsten-rich magmatic fluids migrated and interacted with wall rock, thus forming garnet skarns in the proximal zone and pyroxene skarns in the distal zone (Figure 3). Andradite is characterized by low LREE/HREE ratios and a negative Eu anomaly. The tungsten was incorporated into garnet, reaching high contents of 219-384 ppm (Table 3). The precipitation of garnet with low LREE/HREE ratios likely resulted in an enrichment of LREE and a depletion of HREE in the residual fluids. Sch1a in the proximal zone inherited magmatic-hydrothermal fluids with LREE enrichment, HREE depletion, and negative Eu anomaly (Table 3 and Figure $7 \mathrm{~b}$ ). The tungsten-rich magmatic fluids interacted with the carbonaceous wall rock, which may have led to lower $f \mathrm{O}_{2}$ in the distal Sch1b. The low $f \mathrm{O}_{2}$ can lead to HREE and $Y$ enrichment in scheelite and an alteration in carbonate wall rocks by granite-derived hydrothermal fluids, which release high Sr contents. Sch1b in the distal zone is characterized by an HREE-enriched pattern with lower $\mathrm{MoO}_{3}$ contents, as well as higher $\mathrm{Sr}$ and Y contents (Table 3 and Figure 7d).

Following intensive fluid-rock interaction, the ore-forming fluids showed lower $f \mathrm{O}_{2}$. Replacement of garnet released large amounts of calcium ions into the ore-forming fluid, thus raising the activity of calcium ion in solution, and leading to the dissolution and re-precipitation of Sch1a and Sch1b, as well as the formation of Sch2a and Sch2b $[16,17]$. The lower $f \mathrm{O}_{2}$ resulted in a decrease in $\mathrm{MoO}_{3}$ contents 
in Sch2a. The HREE-depleted patterns of Sch1a and Sch2a were influenced by the precipitation of garnet with low LREE/HREE ratios (Figure 7b). The fluid-rock interaction and alteration in the host rocks by granite-derived hydrothermal fluids can cause Sr enrichment in Sch2a and Sch2b. Sch2a is characterized by HREE-depletion patterns with lower $\mathrm{MoO}_{3}$, and higher Sr contents (Table 3 and Figure $7 \mathrm{~b}$ ). As a result of the overlaying of the late oxidized granodiorite dike to the sample of Sch $2 \mathrm{~b}$ (Figure 3), the ore-forming fluids became higher in $f_{2}$. Sch2b displayed a similar pattern, with granodiorite with higher $\mathrm{MoO}_{3}$ and higher Sr contents (Table 3 and Figure 7d).

The subsequently appearing magmatic-hydrothermal fluids formed quartz-scheelite veins, which typically crosscut early tungsten skarn ores (Figure 5a). The ore-forming fluids increased in $f \mathrm{O}_{2}$ and interacted with the wall rock, thus causing the hydrothermal growth of Sch3. Sch3 exhibits a homogeneous core and growth zoning rim (Figure $5 \mathrm{f}-\mathrm{h}$ ). The homogeneous core of Sch3 displays a similar pattern to that of ore-related granodiorite, which further demonstrates that scheelite is derived from magmatic-hydrothermal fluids. The growth zoning rim displays a strong HREE-depletion pattern, similar to Sch1a and Sch2a, which may be caused by the precipitation of HREE-rich hydrothermal minerals. Continuing fluid-rock interaction can cause Sr enrichment. Sch3 is characterized by LREE-enrichment patterns with higher $\mathrm{MoO}_{3}$ contents, higher REE, Sr, and Y contents compared with Sch2a (Table 3 and Figure 7f). Mixing with meteoric water resulted in the recrystallization of Sch3 and formation of Sch4. Meteoric waters normally show very low contents of REE and trace elements, which could cause a decrease in REE and trace element contents of ore-forming fluid as a result of dilution. Sch4 has a similar pattern to Sch3, but has lower REE, Mo, Sr, and Y contents (Table 3 and Figure $7 \mathrm{~h}$ ). In addition, the hydrogen-oxygen isotope data from both the Jitoushan and Dongyuan deposits (which are closely situated to the Xiaoyao deposit) further demonstrate that the ore-forming fluids are mainly magmatic with the addition of meteoric water during the late stage [51,52].

In summary, textural and trace element signatures indicate a magmatic-hydrothermal formation model and the mechanism of tungsten ore enrichment. Fluid-rock interaction and fluid mixing may be important enrichment mechanisms that generated the Xiaoyao tungsten deposit.

\section{Conclusions}

(1) Scheelite can record the following complex growth histories based on CL images: homogeneous Sch1a and Sch1b, dissolution and re-precipitation Sch2a and Sch2b, oscillatory zoning Sch3, and Sch4 in micro-fractures. These four types of scheelite have different $\mathrm{MoO}_{3}$ and $\mathrm{Cd}$ contents, indicating fluctuating changes in the oxygen fugacity from the skarn stage to the quartz vein stage;

(2) Variations in REE patterns in scheelite are influenced by the REE patterns of ore-related granodiorite, the precipitation of hydrothermal minerals with low LREE/HREE ratios, and changing oxygen fugacity during fluid-rock interaction;

(3) The increasing Sr contents and $\mathrm{Y} /$ Ho ratios of scheelite from the skarn stage to the quartz vein stage indicate continuing fluid-rock interaction. This fluid-rock interaction may be an important enrichment mechanism underlying the generation the Xiaoyao tungsten deposit.

Author Contributions: Q.S. contributed significantly to the data analyses and wrote the manuscript. J.M. conceived and designed the experiment and study design. J.S. helped improve discussions. L.Z. helped perform the trace element analyses experiment. S.X. provided very valuable help during field work. All authors have read and agreed to the published version of the manuscript.

Funding: National Nature Science Foundation of China (No. 41430314).

Acknowledgments: We are grateful to thank the editors and two anonymous reviewers for their critical and constructive comments and suggestions, which helped to improve the paper. We thank Xie Guiqing for his important help to improve the discussion. We are very grateful to Franco Pirajno to polish the language. We would like to thank Wen Wangfei, Fu Jianzhen, and their co-workers from the Anhui Bureau of Geology, Mineral Resources, Exploration and Development, and its affiliated No.332 Geological Team, for support and field help.

Conflicts of Interest: The authors declare no conflicts of interest. 


\section{References}

1. Allen, C.C.; Folinsbee, R.E. Scheelite veins related to porphyry intrusives Hollinger Mine. Econ. Geol. 1944, 39, 340-348. [CrossRef]

2. Noble, S.; Spooner, E.T.; Harris, F. The Logtung large tonnage, low-grade W (scheelite)-Mo porphyry deposit, south-central Yukon Territory. Econ. Geol. 1984, 79, 848-868. [CrossRef]

3. Brugger, J.; Bettiol, A.; Costa, S.; Lahaye, Y.; Bateman, R.; Lambert, D.; Jamieson, D. Mapping REE distribution in scheelite using luminescence. Mineral. Mag. 2000, 64, 891-903. [CrossRef]

4. Hazarika, P.; Mishra, B.; Pruseth, K.L. Scheelite, apatite, calcite and tourmaline compositions from the late Archean Hutti orogenic gold deposit: Implications for analogous two stage ore fluids. Ore Geol. Rev. 2016, 72, 989-1003. [CrossRef]

5. Ghaderi, M.; Palin, J.M.; Campbell, I.H.; Sylvester, P.J. Rare earth element systematics in scheelite from hydrothermal gold deposits in the Kalgoorlie-Norseman region, Western Australia. Econ. Geol. 1999, 94, 423-437. [CrossRef]

6. Dostal, J.; Kontak, D.J.; Chatterjee, A. Trace element geochemistry of scheelite and rutile from metaturbidite-hosted quartz vein gold deposits, Meguma Terrane, Nova Scotia, Canada, Genetic implications. Mineral. Petrol. 2009, 97, 95-109. [CrossRef]

7. Zhang, Z.Y.; Xie, G.Q.; Mao, J.W.; Liu, W.G.; Olin, P.; Li, W. Sm-Nd dating and in-situ LA-ICP-MS trace element analyses of scheelite from the Longshan $\mathrm{Sb}-\mathrm{Au}$ deposit, Xiangzhong metallogenic Province, South China. Minerals 2019, 9, 87. [CrossRef]

8. Zhu, L.Y.; Jiang, S.Y.; Chen, R.S.; Ma, Y. Origin of the Shangfang Tungsten deposit in the Fujian Province of Southeast China: Evidence from scheelite $\mathrm{Sm}-\mathrm{Nd}$ geochronology, $\mathrm{H}-\mathrm{O}$ isotopes and fluid inclusions studies. Minerals 2019, 9, 713. [CrossRef]

9. Song, G.X.; Qin, K.Z.; Li, G.M.; Evans, N.J.; Chen, L. Scheelite elemental and isotopic signatures: Implications for the genesis of skarn-type W-Mo deposits in the Chizhou Area, Anhui Province, Eastern China. Am. Mineral. 2014, 99, 303-317. [CrossRef]

10. Poulin, R.S.; McDonald, A.M.; Kontak, D.J.; McClenaghan, M.B. On the relationship between cathodoluminescence and the chemical composition of scheelite from geologically diverse ore-deposit environments. Can. Mineral. 2016, 54, 1147-1173. [CrossRef]

11. Sciuba, M.; Beaudoin, G.; Grzela, D.; Makvandi, S. Trace element composition of scheelite in orogenic gold deposits. Miner. Depos. 2019, 1-24. [CrossRef]

12. Li, J.D.; Li, X.F.; Xiao, R. Multiple-Stage tungsten mineralization in the Silurian Jiepai W skarn deposit, South China: Insights from cathodoluminescence images, trace elements, and fluid inclusions of scheelite. J. Asian Earth Sci. 2019, 181, 1-22. [CrossRef]

13. Yuan, L.L.; Chi, G.X.; Wang, M.Q.; Li, Z.H.; Xu, D.R.; Deng, T.; Geng, J.Z.; Hu, MY.; Zhang, L. Characteristics of REEs and trace elements in scheelite from the Zhuxi $\mathrm{W}$ deposit, South China: Implications for the ore-forming conditions and processes. Ore Geol. Rev. 2019, 109, 585-597. [CrossRef]

14. Wu, S.H.; Mao, J.W.; Ireland, T.R.; Zhao, Z.; Yao, F.J.; Yang, Y.P.; Sun, W.D. Comparative geochemical study of scheelite from the Shizhuyuan and Xianglushan tungsten skarn deposits, South China: Implications for scheelite mineralization. Ore Geol. Rev. 2019, 109, 448-464. [CrossRef]

15. Sun, K.K.; Chen, B.; Deng, J. Ore genesis of the Zhuxi supergiant W-Cu skarn polymetallic deposit, South China: Evidence from scheelite geochemistry. Ore Geol. Rev. 2019, 107, 14-29. [CrossRef]

16. Kwak, T.A.P.; Tan, T.H. The geochemistry of zoning in skarn minerals at the King Island (Dolphin) mine. Econ. Geol. 1981, 76, 468-497. [CrossRef]

17. Newberry, R.J. W-and Sn-Skarn Deposits: A 1998 Status Report. Miner. Intrusion-Relat. skarn Syst. 1998, 26, 289-335.

18. Meinert, L.D.; Dipple, G.M.; Nicolescu, S. World skarn deposits. Econ. Geol. 2005, 100, 299-336.

19. Wang, D.E.; Zhou, X.; Yu, X.Q.; Du, Y.D.; Yang, H.M.; Fu, J.Z.; Dong, H.M. SHRIMP zircon U-Pb dating and characteristics of Hf isotopes of the granodiorite porphyries in the Dongyuan W-Mo ore district, Qimen area, southern Anhui. Geol. Bull. China 2011, 30, 1514-1529. (In Chinese)

20. Xiang, X.K.; Wang, P.; Sun, D.M.; Zhong, B. Re-Os isotopic age of molybdeinte from the Shimensi tungsten polymetallic deposit in northern Jiangxi province and its geological implications. Geol. Bull. China 2013, 32, 1824-1831. (In Chinese) 
21. Mao, J.W.; Xiong, B.K.; Liu, J.; Pirajno, F.; Cheng, Y.B.; Ye, H.S.; Song, S.W.; Dai, P. Molybdenite Re/Os dating, zircon $\mathrm{U}-\mathrm{Pb}$ age and geochemistry of granitoids in the Yangchuling porphyry W-Mo deposit (Jiangnan tungsten ore belt), China: Implications for petrogenesis, mineralization and geodynamic setting. Lithos 2017, 286-287, 35-52. [CrossRef]

22. Mao, Z.H.; Cheng, Y.B.; Liu, J.J.; Yuan, S.D.; Wu, S.H.; Xiang, X.K.; Luo, X.H. Geology and molybdenite Re-Os age of the Dahutang granite-related veinlets-disseminated tungsten ore field in the Jiangxin Province, China. Ore Geol. Rev. 2013, 53, 422-433. [CrossRef]

23. Chen, G.H.; Shu, L.S.; Shu, L.M.; Zhang, C.; Ouyang, Y.P. Geological characteristics and mineralization setting of the Zhuxi tungsten (copper) polymetallic deposit in the Eastern Jiangnan Orogen. Sci. China Earth Sci. 2015, 45, 1799-1818. (In Chinese) [CrossRef]

24. Mao, Z.H.; Liu, J.J.; Mao, J.W.; Deng, J.; Zhang, F.; Meng, X.Y.; Xiong, B.K.; Xiang, X.K.; Luo, X.H. Geochronology and geochemistry of granitoids related to the giant Dahutang tungsten polymetallic deposit, middle Yangtze River region, China: Implications for petrogenesis, geodynamic setting, and mineralization. Gondwana Res. 2015, 28, 816-836. [CrossRef]

25. Song, S.W.; Mao, J.W.; Xie, G.Q.; Chen, L.; Santosh, M.; Chen, G.H.; Rao, J.F.; Ouyang, Y.P. In situ LA-ICP-MS $\mathrm{U}-\mathrm{Pb}$ geochronology and trace element analysis of hydrothermal titanite from the giant Zhuxi W $(\mathrm{Cu})$ skarn deposit, South China. Miner. Depos. 2019, 54, 569-590. [CrossRef]

26. Song, S.W.; Mao, J.W.; Zhu, Y.F.; Yao, Z.Y.; Chen, G.H.; Rao, J.F.; Ouyang, Y.P. Partial-Melting of fertile metasedimentary rocks controlling the ore formation in the Jiangnan porphyry-skarn tungsten belt, South China: A upcletn, case study at the giant Zhuxi W-Cu skarn deposit. Lithos 2018, 304, 180-199. [CrossRef]

27. Team, No. 332. Exploration Report of the Xiaoyao Tungsten Polymetallic Deposit, Jixi County, Anhui Province; Anhui Bureau of Geology and Mineral Resources: Anhui, China, 2011.

28. Su, Q.W.; Mao, J.W.; Wu, S.H.; Zhang, Z.C.; Xu, S.F. Geochronology and geochemistry of the granitoids and ore-forming age in the Xiaoyao tungsten polymetallic skarn deposit in the Jiangnan Massif tungsten belt, China: Implications for their petrogenesis, geodynamic setting, and mineralization. Lithos 2018, 296-299, 365-381. [CrossRef]

29. Chen, F.; Wang, D.H.; Du, J.G.; Xu, W.; Hu, H.F.; Yu, Y.L.; Tang, J.L. New dating of the fuling granite body with LA-ICP-MS zircon U-Pb in Jixi, Anhui Province and their geological significance. Rock Miner. Anal. 2013, 32, 970-977. (In Chinese)

30. Sun, K.K.; Chen, B. Trace elements and Sr-Nd isotopes of scheelite: Implications for the W-Cu-Mo polymetallic mineralization of the Shimensi deposit, South China. Am. Mineral. 2017, 102, 1114-1128.

31. Zaw, K.; Singoyi, B. Formation of magnetite-scheelite skarn mineralization at Kara, Northwestern Tasmania: Evidence from mineral chemistry and stable Isotopes. Econ. Geol. 2000, 95, 1215-1230. [CrossRef]

32. Timon, S.S.M.; Moro, B.M.C.; Cembranos, P.M.L. Mineralogical and physiochemical evolution of the Los Santos scheelite skarn, Salamanca, NW Spain. Econ. Geol. 2009, 104, 961-995.

33. Sun, S.S.; Mcdonough, W.F. Chemical and isotopic systematics of oceanic basalts: Implications for mantle composition and processes. Geol. Soc. Lond. Spec. Publ. 1989, 42, 313-345. [CrossRef]

34. Hsu, L.C.; Galli, P.E. Origin of the scheelite-powellite series of minerals. Econ. Geol. 1973, 68, 681-696. [CrossRef]

35. Rempel, K.U.; Williams-Jones, A.E.; Migdisov, A.A. The partitioning of molybdenum (VI) between aqueous liquid and vapour at temperatures up to $370{ }^{\circ} \mathrm{C}$. Geochim. Cosmochim. Acta 2009, 73, 3381-3392. [CrossRef]

36. Xu, J.; Ciobanu, C.L.; Cook, N.J.; Slattery, A. Crystals from the powellite-scheelite series at the nanoscale: A case study from the Zhibula Cu Skarn, Gangdese Belt, Tibet. Minerals 2019, 9, 340. [CrossRef]

37. Liu, Y.; Cao, L.; Li, Z.; Wang, H.; Chu, T.; Zhang, J. Element Geochemistry; Science Press: Beijing, China, 1984; p. 548.

38. Liu, B.; Li, H.; Wu, Q.H.; Evans, N.J.; Cao, J.Y.; Jiang, J.B.; Wu, J.H. Fluid evolution of Triassic and Jurassic W mineralization in the Xitian ore field, South China: Constraints from scheelite geochemistry and microthermometry. Lithos 2019, 330-331, 1-15. [CrossRef]

39. Guo, S.; Chen, Y.; Liu, C.; Wang, J.; Su, B.; Gao, Y.; Wu, F.; Sein, K.; Yang, Y.; Mao, Q. Scheelite and coexisting F-rich zoned garnet, vesuvianite, fluorite, and apatite in calc-silicate rocks from the Mogok metamorphic belt, Myanmar: Implications for metasomatism in marble and the role of halogens in $\mathrm{W}$ mobilization and mineralization. J. Asian Earth Sci. 2016, 117, 82-106. [CrossRef] 
40. Poulin, R.S.; Kontak, D.J.; McDonald, A.; Beth, M.; McCLenaghan, B.M. Assessing Scheelite as an ore-deposit discriminator using its trace-element REE chemistry. Can. Mineral. 2018, 56, 265-302. [CrossRef]

41. Ismail, R.; Ciobanu, C.; Cook, N.J.; Teale, G.S.; Giles, D.; Mumm, A.S.; Wade, B. Rare earths and other trace elements in minerals from skarn assemblages, Hillside iron oxide-copper-gold deposit, Yorke Peninsula, South Australia. Lithos 2014, 184-187, 456-477. [CrossRef]

42. Kinsman, D.J.J. Interpretation of $\mathrm{Sr}^{2+}$ concentrations in carbonate minerals and rocks. J. Sediment. Petrol. 1969, 39, 486-508.

43. Friedmann, I.; O'Neil, J.R. Compilation of stable isotope fractionation factors of geochemical interest: Chapter KK. In Data of Geochemistry, 6th ed.; Fleischer, M., Ed.; U.S. Geological Survey Professional Paper; U.S. Government Printing Office: Washington, DC, USA, 1977; Volume 440, p. 12.

44. Lecumberri-Sanchez, P.; Vieira, R.; Heinrich, C.A.; Pinto, F.; Wälle, M. Fluid-Rock interaction is decisive for the formation of tungsten deposits. Geology 2017, 45, 579-582. [CrossRef]

45. Kozlik, M.; Gerdes, A.; Raith, J.G. Strontium isotope systematics of scheelite and apatite from the Felbertal tungsten deposit, Austria-results of in-situ LA-MC-ICP-MS analysis. Miner. Pet. 2016, 110, 11-27. [CrossRef]

46. Burt, D.M. Compositional and phase relations among rare earth elements. Rev. Mineral. 1989, 21, $259-307$.

47. Schmidt, M.W.; Dardon, A.; Chazot, G.; Vannucci, R. The dependence of $\mathrm{Nb}$ and Ta rutile-melt partitioning on melt composition and $\mathrm{Nb} / \mathrm{Ta}$ fractionation during subduction processes. Earth Planet Sci. Lett. 2004, 226, 415-432. [CrossRef]

48. Yaxley, G.M.; Green, D.H.; Kamenetsky, V. Carbonatite metasomatism in the southeastern Australian lithosphere. J. Petrol. 1998, 39, 1917-1930. [CrossRef]

49. Taylor, S.R.; McLennan, S.M. The Continental Crust: Its Composition and Evolution; Blackwell: Oxford, UK, 1985; p. 312.

50. Dostal, J.; Chatterjee, A.K. Contrasting behaviour of $\mathrm{Nb} / \mathrm{Ta}$ and $\mathrm{Zr} / \mathrm{Hf}$ ratios in a peraluminous granitic pluton (Nova Scotia, Canada). Chem. Geol. 2000, 123, 67-88. [CrossRef]

51. Du, Y.D.; Liu, J.J.; Yu, X.Q.; Zhou, X.; Yang, H.M.; Yang, L.B.; Huang, Y.H. The sources of metallogenic materials and mineralization of the Xiaoyao $\mathrm{W}$ polymetallic deposit in Anhui Province: Evidence from carbon, sulfur and lead isotopes. Geol. China 2013, 40, 566-579. (In Chinese)

52. Song, G.X.; Qin, K.Z.; Li, G.M.; Li, X.H.; Qu, W.J. Geochronology and ore-forming fluids of the Baizhangyan W-Mo deposit in the Chizhou area, middle-lower Yangtze valley, SE-China. Resour. Geol. 2012, 63, 57-71. [CrossRef]

(C) 2020 by the authors. Licensee MDPI, Basel, Switzerland. This article is an open access article distributed under the terms and conditions of the Creative Commons Attribution (CC BY) license (http://creativecommons.org/licenses/by/4.0/). 